\title{
A new construction of antipodal distance regular covers of complete graphs through the use of Godsil-Hensel matrices
}

\author{
Mikhail Klin \\ Department of Mathematics, Ben-Gurion University of the Negev \\ 84105 Beer Sheva, Israel \\ Christian Pech * \\ Lößnitzgrundstr. 47, 01445 Radebeul, Germany
}

Received 10 December 2009, accepted 14 February 2011, published online 15 May 2011

\begin{abstract}
New constructions of regular distance regular antipodal covers (in the sense of GodsilHensel) of complete graphs $K_{n}$ are presented. The main source of these constructions are skew generalized Hadamard matrices. It is described how to produce such a matrix of order $n^{2}$ over a group $T$ from an arbitrary given generalized Hadamard matrix of order $n$ over the same group $T$. Further, a new regular cover of $K_{45}$ on 135 vertices is produced with the aid of a decoration of the alternating group $A_{6}$.

Keywords: Antipodal graph, automorphism group, association scheme, conference matrix, distance regular cover, generalized Hadamard matrix, Godsil-Hensel matrix, group ring, Foster graph, Mathieu group, Payne's doily, resolvable transversal design, Schur multiplier, semibiplane, Taylor graph, two-graph, Tutte's 8-cage, Wielandt's 2-closure.
\end{abstract}

Math. Subj. Class.: 05E30, 05B20, 20B25, 05E18

\section{Introduction}

Antipodal covers of complete graphs $K_{n}$ form a significant particular case of imprimitive distance regular graphs. Distance transitive antipodal covers of $K_{n}$ were classified in [46]. The investigation of all antipodal distance regular covers of $K_{n}$ has been the subject of numerous publications during the last two decades. In [45], special attention was paid to

\footnotetext{
* Supported by the Skirball postdoctoral fellowship of the Center of Advanced Studies in Mathematics at the Mathematics Department of the Ben-Gurion University of the Negev in Beer Sheva, Israel

E-mail addresses: klin@cs.bgu.ac.il (Mikhail Klin), cpech@ freenet.de (Christian Pech)
} 
regular covers of complete graphs. Such covers are distinguished from the general antipodal distance regular covers of $K_{n}$ by the property that the stabilizer $T$ of all fibres in the full automorphism group acts regularly on each fibre. As a result, every regular cover may be described with the aid of a suitable matrix over $\mathbb{Z}[T]$ (the Godsil-Hensel matrix of the regular cover).

In the present paper we set forth new constructions of antipodal covers of $K_{n}$ via the use of new classes of Godsil-Hensel matrices. The main source is provided by generalized Hadamard matrices.

Sections 2 and 3 contain all necessary preliminaries about distance regular covers and antipodal covers of $K_{n}$ in particular. Regular covers and Godsil-Hensel matrices are introduced in Section 4.

In Section 5, after considering the concept of a generalized Hadamard matrix $\operatorname{gH}(T, n)$ of order $n$ over a group $T$, we create a link between Godsil-Hensel matrices and generalized Hadamard matrices (cf. Lemma 5.3, Corollary 5.4). This establishes the methodological background of the further results. The crucial Theorem 5.6 provides a way to construct from an arbitrary $\mathrm{gH}(T, n)$ a new skew $\mathrm{gH}\left(T, n^{2}\right)$. According to Corollary 5.4, such skew matrices imply the existence of antipodal, regular, distance regular covers of $K_{n}$. A number of striking new examples of regular covers resulting from small generalized Hadamard matrices are considered in Section 6.

Section 7 is concerned with a new stand alone construction of a regular antipodal cover on 135 vertices. The corresponding Godsil-Hensel matrix is produced from the Foster graph on 90 vertices (the unique 3 -fold antipodal distance regular cover of Tutte's 8-cage on 30 vertices).

Sections 8 and 9 introduce further new byproduct results and provide a summary of new constructions in comparison with the known antipodal covers.

The concluding (and quite comprehensive) Section 10 is written in a different style (compared with the main body of the paper). It gives a digest of interdisciplinary links between the antipodal covers and other diverse combinatorial structures. It serves to bring the reader to the forefront of possible further generalizations and applications of the results presented in this paper.

We assume that the reader is familiar with the elementary background concepts from the theory of association schemes, and distance regular graphs. In particular such sources as [10], [30], [69] may provide a helpful assistance.

Many of the presented results rely on the essential use of computer packages and separate programs, such as [29], [35], [100], [78]. The recent paper [71] provides a reasonable discussion of the abilities of computer algebra experimentation in algebraic graph theory.

\section{Distance regular covers}

Let us quickly recall the definitions of the concepts that appear in the title of this section. For more details we refer to [10]. A graph $\Gamma$ for us will be a pair $(V, E)$ where $V=V(\Gamma)$ is a finite set of vertices and $E=E(\Gamma) \subseteq\left(\begin{array}{l}V \\ 2\end{array}\right)$ is a set of undirected edges.

Recall that a graph $\Gamma$ is called a distance regular graph (DRG) if it is connected and if for any two vertices the number of vertices of distance $i$ from the first and distance $j$ from the second depends only on $i, j$, and the distance $k$ between the two initial vertices. This number is denoted by $p_{i, j}^{k}$. If we define binary relations $R_{i}$ on $V(\Gamma)$ by putting a pair of vertices into $R_{i}$ whenever their distance in $\Gamma$ equals to $i$, then all these distance 
relations form an association scheme. If this scheme is primitive (all distance relations are connected) then $\Gamma$ is called primitive DRG, otherwise $\Gamma$ is called imprimitive. If $d$ is the diameter of $\Gamma$ and if $R_{d}$ is an equivalence relation, then $\Gamma$ is called antipodal. The relation $R_{d}$ is called the antipodality relation of the DRG.

A graph $\Gamma$ is called a cover of another graph $\Delta$ is there is a surjection $h: V(\Gamma) \rightarrow$ $V(\Delta)$ that maps edges of $\Gamma$ to edges of $\Delta$ and that has the additional property to be locally an isomorphism - that is if we restrict $h$ to the set $\mathrm{U}$ of neighbors of any vertex $u \in V(\Gamma)$, then $\left.h\right|_{U}: U \rightarrow U^{h}$ is an isomorphism ${ }^{1}$. The function $h$ is called a covering of $\Delta$ and the preimages of vertices from $\Delta$ are called the fibres of the covering. Clearly, each fibre induces an empty subgraph and between two fibres there are either no edges or the edges between the fibres form a perfect matching. In particular for covers of connected graphs the fibres are all of the same size $r$. This number is commonly called the index of the cover. If a cover $\Gamma$ of $\Delta$ is induced by a covering $h$, and if $\operatorname{ker}(h)$ is the equivalence relation of $V(\Gamma)$ corresponding to the partition into fibres of $h$, then we can define $\Gamma / \operatorname{ker}(h)$ as follows. The vertices are the fibres and two fibres are connected by an edge if there is a matching between them in $\Gamma$. A basic observation is that $\Gamma / \operatorname{ker}(h)$ is isomorphic to $\Delta$. If an equivalence relation on $V(\Gamma)$ arises as the kernel of a covering, then we call it a covering relation. Covering relations can easily be axiomatized independently: an equivalence relation $\theta$ on $V(\Gamma)$ is a covering relation if and only if its equivalence classes are independent sets in $\Gamma$ and between two equivalence classes there is either no edge or a perfect matching in $\Gamma$.

If the cover $\Gamma$ of a graph $\Delta$ turns out to be distance regular, then we speak about a distance regular cover.

It is well known that imprimitive DRGs are bipartite, antipodal, or both. Moreover, if a DRG is antipodal then the antipodality relation is a covering relation, moreover, the quotient graph by the antipodality relation is a DRG that is not antipodal and that is bipartite if and only if the original DRG was bipartite. Covers induced by antipodality relations are called antipodal covers.

The thesis [66] is an excellent source for further information about antipodal covers of DRGs.

\section{Antipodal covers of $K_{n}$}

A first attempt of a systematization of the research about distance regular graphs in general and about antipodal DRGs in particular was accomplished in [10]. The paper [45] is regarded by us as the next significant cornerstone in the study about antipodal distance regular covers of complete graphs with the hope to get from these covers a more general understanding of antipodal DRGs. Another motivation for studying antipodal covers of complete graphs is their close relation to certain geometric structures such as group divisible designs, projective planes, and generalized quadrangles.

We consider the whole system of facts about antipodal covers, which is presented in [45], as the Godsil-Hensel classification scheme (note however, that a part of the results presented in it is, as it is stressed by the authors, simply borrowed from predecessors).

The first step is a parametrization of antipodal covers of $K_{n}$. According to [45, Lem. 3.1] an arbitrary $r$-fold cover of $K_{n}$ is antipodal distance regular if and only if there exists a constant $c_{2}$ such that any two non-adjacent vertices from different fibres of the cover have

\footnotetext{
$U$.

${ }^{1}$ Here we slightly abuse notation by identifying a subset $U$ of vertices of $\Gamma$ with the subgraph of $\Gamma$ induced by
} 
exactly $c_{2}$ common neighbors. Thus, $r$-fold antipodal distance regular covers of $K_{n}$ can be parametrized by a triple $\left(n, r, c_{2}\right)$. To save space we will call an antipodal distance regular cover of $K_{n}$ with parameters $\left(n, r, c_{2}\right)$ simply an $\left(n, r, c_{2}\right)$-cover.

The basic feasibility conditions for such triples are the following (cf. [45]):

(F1) $n, r, c_{2}$ are integers with $1 \leq(r-1) c_{2} \leq n-2$,

(F2) if $n$ is even then $c_{2}$ is even,

(F3) the multiplicities of the eigenvalues of the cover are integers.

For completeness we recall the formulae for the eigenvalues and their respective multiplicities of the $\left(n, r, c_{2}\right)$-covers: If $\delta=n-2-r c_{2}, \Delta=\delta^{2}+4(n-1)$, then the eigenvalues are

$$
n-1, \quad-1, \quad \theta=\frac{\delta+\sqrt{\Delta}}{2}, \quad \tau=\frac{\delta-\sqrt{\Delta}}{2} .
$$

Here $n-1$ has multiplicity 1 , and the eigenvalue -1 has multiplicity $n-1$. The multiplicities of $\theta$ and $\tau$ are given by

$$
m_{\theta}=\frac{n(r-1) \tau}{\tau-\theta}, \quad m_{\tau}=\frac{n(r-1) \theta}{\theta-\tau}, \text { respectively }
$$

The next step in the classification is the division of $\left(n, r, c_{2}\right)$-covers into four parameter classes which is based on the following theorem:

Theorem 3.1 ([45, Thm. 3.6]). For fixed $r$ and $\delta$ there are only finitely many feasible parameter triples, unless $\delta \in\{-2,0,2\}$.

Thus four natural classes of parameter triples may be distinguished according to the value of $\delta$ :

$$
\begin{aligned}
& \text { I } \delta=-2, \\
& \text { II } \delta=0 \text { (standard cover in the sense of [44]), } \\
& \text { III } \delta=2, \\
& \text { IV } \delta \notin\{-2,0,2\} .
\end{aligned}
$$

An important consequence from the basic feasibility conditions is that if $\delta \in\{-2,2\}$, then $n$ must be a square (cf. [45, Lem. 3.7])

In Table 1 below we sum up the constructions of infinite series of distance regular antipodal covers of $K_{n}$, that are known to us: 
M. Klin and C. Pech: A new construction of antipodal distance regular covers...

\begin{tabular}{|l|c|l|c|l|} 
construction & parameters & conditions & class & references \\
\hline \hline Mathon & $(q+1, r, c)$ & $\begin{array}{l}q=r c+1 \text { prime } \\
\text { power }\end{array}$ & II & [76], [10, 12.5.3] \\
\hline Cameron & $\left(q^{3}+1, r,\left(q^{3}-1\right) / r\right)$ & $\begin{array}{l}q \text { prime power, } r \\
\text { odd, } r \mid(q-1)\end{array}$ & II & {$[14]$} \\
\hline Bondy & $(n, n-2,1)$ & $\begin{array}{l}\text { a projective plane } \\
\text { of order } n-1 \text { ex- } \\
\text { ists }\end{array}$ & II & $\begin{array}{l}{[5],} \\
{[45,4.2]}\end{array}$ \\
\hline Thas-Somma & $\left(q^{2 j}, q, q^{2 j-1}\right)$ & $\begin{array}{l}q \text { prime power } \\
\text { Brouwer }\end{array}$ & I & $\begin{array}{l}{[107],[101],[10,} \\
12.5 .1],[45,4.3]\end{array}$ \\
\hline Godsil-Hensel & $\left(p^{2 i}, p^{i-k}, p^{i+k}\right)$ & $\begin{array}{l}p \text { prime, } \\
0 \leq k<i\end{array}$ & I & {$[45,6.4]$} \\
\hline $\begin{array}{l}\text { de Caen-Mathon- } \\
\text { Moorhouse }\end{array}$ & $\left(2^{2 t}, 2^{2 t-1}, 2\right)$ & & I & {$[24],[3]$} \\
\hline $\begin{array}{l}\text { de Caen and } \\
\text { Fon-Der-Flaass }\end{array}$ & $\left(q^{d+1}, q^{d}, q\right)$ & $q=2^{t}$ & I & {$[23]$} \\
\hline
\end{tabular}

Table 1: Known infinite classes of distance regular covers of complete graphs.

Remark 3.2. i) The Cameron construction works also in certain cases where $r \mid(q+1)$ and $r$ is not necessarily odd.

ii) The Godsil-Hensel construction is an extension of the Thas-Somma construction using a quotient construction given in [45, Cor. 6.3].

iii) Combining the quotient construction with the de Caen-Mathon-Moorhouse construction, covers with parameters $\left(2^{2 t}, 2^{2 t-i}, 2^{i}\right)$ for $1 \leq i \leq 2 t$ can be obtained. All these covers are from class I.

iv) Bending and Fon-Der-Flaass in [3] observed that the de Caen-Mathon-Moorhouse construction can be generalized using so called crooked functions. However, so far the known crooked functions only give the graphs already obtainable from the de CaenMathon-Moorhouse construction.

v) The construction by de Caen and Fon-Der-Flaass is a generalization of the de CaenMathon-Moorhouse construction.

vi) Combining the quotient construction with the de Caen and Fon-Der-Flaass construction, covers with parameters $\left(2^{t(d+1)}, 2^{t d-k}, 2^{t+k}\right)$ may be obtained. All these covers are from class I.

vii) Godsil, Liebler, and Praeger in [46] characterized all distance transitive covers of $K_{n}$. They refer to the unpublished manuscript [11], however do not express in evident form the parameters of the considered series. We did not investigate, whether Table 1 indeed

\footnotetext{
${ }^{3}$ This denotes a strongly regular graph (briefly SRG) which has the parameters of the point graph of a generalized quadrangle of order $(s, t)$.
} 
covers all known infinite series of parameters of antipodal distance regular covers of complete graphs.

\section{Regular covers}

Let $\Gamma$ be a connected cover of some graph $\Delta$ and consider the group $T$ of all automorphisms of $\Gamma$ that fix each fibre of $\Gamma$ setwise. Then $T$ acts semi-regularly on $V(\Gamma)$ (cf. [45, Sec. 7]), and in particular on each fibre of $\Gamma$. If $T$ acts regularly on each fibre, then $\Gamma$ is called a regular cover of $\Delta$. We will call the group $T$ the voltage group of the cover (cf. [49]).

Godsil and Hensel in [45] studied regular covers in general and in particular gave a characterization of regular antipodal distance regular covers of complete graphs. For this they used certain matrices over group rings, that we will introduce below (note that our notation will differ from the one in [45]).

\subsection{Matrices over group rings}

For a given finite group $T$, and a given unitary ring $(R,+, \cdot, 0,1)$ we denote by $R[T]$ the group ring of $T$ over $R$. Following the theory of Schur rings (cf. [110]), for a subset $M$ of $T$ the simple quantity $\underline{M} \in R[T]$ is defined by

$$
\underline{M}:=\sum_{m \in M} 1 \cdot m \text {. }
$$

Definition 4.1. With the notions from above, for $n$ a positive natural number, a matrix of order $n$ over $R[T]$ is a function

$$
A:\{1,2 \ldots, n\}^{2} \rightarrow R[T] .
$$

Usually we will denote a matrix $A$ by $\left(a_{i, j}\right)_{i, j=1}^{n}$ where $a_{i, j}=A(i, j)$. Moreover, if the order of $A$ is clear from the context, then we will shorten this notation by writing $A=\left(a_{i, j}\right)$.

Matrices over group rings can be added, subtracted and multiplied in the usual way. The set of all matrices of order $n$ over $R[T]$ will be denoted by $R[T]^{n \times n}$. Together with addition and multiplication this set forms a unital ring. Moreover, the group ring $R[T]$ acts naturally from the right (and from the left) on $R[T]^{n \times n}$.

If $e$ is the neutral element of $T$ then by $I$ we denote the matrix that has everywhere on the diagonal the element $\underline{e}$ and that is zero outside of the diagonal. Moreover we define $J$ to be the matrix that is everywhere equal to $\underline{e}$ (do not confuse the multiplicative identity element $\underline{e}$ with the additive identity element 0 of $R[T]$ ).

Finally, we can equip $R[T]^{n \times n}$ with a unary operation of adjunction by extending the operation of inversion of $T$ first to $R[T]$ in the natural way by $\left(\sum a_{g} g\right)^{*}:=\sum a_{g} g^{-1}$ and then to define for a matrix $A=\left(a_{i, j}\right)$

$$
A^{*}=\left(a_{i, j}\right)^{*}:=\left(a_{j, i}^{*}\right) .
$$

If $A=A^{*}$, then we call $A$ self-adjoint.

\subsection{Godsil-Hensel matrices}

Following we will describe how regular covers with voltage group $T$ in general can be obtained from certain matrices over the integral group ring of $T$. Such a characterization 
was given for the first time in [45] as a matrix representation of so called symmetric arc functions. This is the reason why we like to call the matrix that gives rise to a given cover the Godsil-Hensel matrix of this cover.

Let $T$ be a finite group and let $A=\left(a_{i, j}\right) \in \mathbb{Z}[T]^{n \times n}$ such that $a_{i, j} \in(\{g \mid g \in$ $T\} \cup\{0\}$ ), all elements on the diagonal are equal to 0 , and such that $A$ is self-adjoint. Then to $A$ we can associate two graphs:

1. The underlying graph $\Delta_{A}$ with vertex set $V\left(\Delta_{A}\right)=\{1,2, \ldots, n\}$ and edge set $E\left(\Delta_{A}\right)=\left\{\{i, j\} \mid a_{i, j} \neq 0\right\}$,

2. The derived graph $\Gamma^{A}$ with vertex set $V\left(\Gamma^{A}\right)=\{1,2, \ldots, n\} \times T$ and edge set $E\left(\Gamma^{A}\right)=\left\{\{(i, g),(j, h)\} \mid a_{i, j} \neq 0\right.$, and $\left.\underline{g} \cdot a_{i, j}=\underline{h}\right\}$.

It is not hard to see that $\Gamma^{A}$ is a cover of $\Delta_{A}$. Indeed, the function $f: V\left(\Gamma^{A}\right) \rightarrow V\left(\Delta_{A}\right)$ that is defined by $(i, g) \mapsto i$ is a covering. Moreover, each cover with voltage group $T$ can be obtained in this way, up to isomorphism.

However, there are matrices $A$ that define covers whose voltage group is not equal to $T$.

From now on we will work only with such matrices over $\mathbb{Z}[T]$ that define connected covers with voltage group $T$. Such matrices we will call covering matrices.

In $[45$, Lem. 7.1, 7.2] covering matrices are characterized. Here we only give the special case when $\Delta_{A}$ is a complete graph. In this case only the diagonal elements of $A$ are equal to 0 . If $A=\left(a_{i, j}\right)_{i, j=1}^{n}$, then $A$ is a covering matrix if and only if $T$ is generated by the set

$$
\left\{a_{i, 1}^{-1} \cdot a_{i, j} \cdot a_{1, j}^{-1} \mid i \neq j, 1 \leq i, j \leq n\right\} .
$$

If for a covering matrix $A$ the graph $\Gamma^{A}$ is an antipodal distance regular cover of $\Delta_{A}$, then we call $A$ the Godsil-Hensel matrix of this cover.

Theorem 4.2 ([45, Cor. 7.5]). Let $T$ be a finite group and let $A$ be a covering matrix 4 of order $n$ over $T$. Then $A$ is the Godsil-Hensel matrix of a regular antipodal $\left(n, r, c_{2}\right)$-cover of $K_{n}$ with voltage group $T$ if and only if

$$
A^{2}=(n-1) I+\delta A+c_{2} \underline{T}(J-I), \quad \text { where as before } \delta=n-2-r c_{2} .
$$

The collection of all such matrices $A$ will be denoted by $\operatorname{GHM}\left(T, n, r, c_{2}\right)$.

\section{Distance regular antipodal covers from generalized Hadamard ma- trices}

In this section we will derive a new construction of regular antipodal distance regular covers of complete graphs. In order to describe it, we need a few more notions.

Definition 5.1. Let $T$ be a finite group and let $A=\left(a_{i, j}\right) \in \mathbb{Z}[T]^{n \times n}$ such that $a_{i, j} \in\{\underline{g} \mid$ $g \in T\}$ for all $i$ and $j$. Then $A$ is called a generalized Hadamard matrix if for $c=\frac{n}{|T|}$, we have that

$$
A A^{*}=A^{*} A=n I+c \underline{T}(J-I) .
$$

The set of all generalized Hadamard matrices of order $n$ over $T$ will be denoted by $\operatorname{gH}(T, n)$.

\footnotetext{
${ }^{4}$ Note that covering matrices are always self-adjoint and have a 0-diagonal.
} 
Remark 5.2. i) There are several natural ways to generalize the concept of Hadamard matrices. In this paper we use the generalization due to Drake [28]. See Section 10.7 for more details. The various kinds of generalized Hadamard matrices are also surveyed in [55, Chap. 4].

ii) In the definition of generalized Hadamard matrices the condition $A A^{*}=A^{*} A$ is redundant (cf. [21, p. 457]).

iii) Generalized Hadamard matrices $\mathrm{gH}(T, n)$ are special cases of difference matrices with parameters $(|T|, n ; n /|T|)$ (cf. [4, Def. VIII.3.4]). In turn, difference matrices are in a one to one correspondence with so called class-regular resolvable transversal designs (cf. [4, Thm. VIII.3.6]). In particular, every $\mathrm{gH}(T, n)$ gives rise to a class regular $\operatorname{RTD}_{n /|T|}(n,|T|)$. In the particular case, when $n /|T|=2$ this is a special case of so called elation semibiplanes $\operatorname{SBP}(n \cdot|T|, n)$.

It was shown by Shad [94] and Delorme [26] (cf. [95]) that generalized Hadamard matrices of order $n$ characterize the regular antipodal distance regular covers of $K_{n, n}$. Following we will point out a new connection between generalized Hadamard matrices and distance regular antipodal regular covers of complete graphs. First we will give an alternative way to characterize the Godsil-Hensel matrices of such covers:

Lemma 5.3. Let $T$ be a finite group and let $A \in \mathbb{Z}[T]^{n \times n}$ be a covering matrix with $\Delta_{A}=K_{n}$. Then $\Gamma^{A}$ is distance regular if and only if there exist numbers $r$ and $c_{2}$ such that

$$
(A+I)^{2}=n I+\left(n-r c_{2}\right) A+c_{2} \underline{T}(J-I) .
$$

Proof.

$$
\begin{aligned}
(A+I)^{2} & =A^{2}+2 A+I \\
& =(n-1) I+\left(n-2-r c_{2}\right) A+c_{2} \underline{T}(J-I)+2 A+I \\
& =n I+\left(n-r c_{2}\right) A+c_{2} \underline{T}(J-I) .
\end{aligned}
$$

From this formulation of the criterion, the connection of regular $\left(n, r, c_{2}\right)$-covers with generalized Hadamard matrices becomes evident. In particular, if $n-r c_{2}=0$ (i.e., if $\delta=$ $-2)$, then $A+I$ must be a $\mathrm{gH}(T, n)$. So summing up, we get the following characterization of regular $\left(n, r, c_{2}\right)$-covers with $\delta=-2$ (i.e., such covers from family I):

Corollary 5.4. Let $T$ be a finite group with neutral element e, and let $A \in \mathbb{Z}[T]^{n \times n}$ be a covering matrix with $\Delta_{A}=K_{n}$. Then the graph $\Gamma^{A}$ is an antipodal regular cover of $K_{n}$ with $\delta=-2$ if and only if $(A+I)$ is a self-adjoint $\mathrm{gH}(T, n)$ with diagonal entries all equal to $\underline{e}$.

Remark 5.5. i) Self-adjoint generalized Hadamard matrices with identity diagonal are sometimes called skew. We will furthermore adopt this name.

ii) If $T=(\{+1,-1\}, \cdot)$ is the cyclic group of order 2 , then generalized Hadamard matrices over $T$ coincide with classical Hadamard matrices and skew generalized Hadamard matrices over $T$ coincide with symmetric Hadamard matrices with identity diagonal. 
Moreover, all distance regular double covers of $K_{n}$ are regular covers. Hence, we obtain that distance regular antipodal double covers of $K_{n}$ with $\delta=-2$ are equivalent to symmetric Hadamard matrices with identity diagonal.

iii) Distance regular double covers of $K_{n}$ are also known under the name Taylor graphs. It is well known that there is a one-to-one correspondence between Taylor graphs and regular two-graphs (cf. [106]).

iv) Thus symmetric Hadamard matrices with identity diagonal give a rich source of regular two-graphs (see Section 10 for a discussion).

v) The parameters of the regular cover obtained from a skew $\mathrm{gH}(T, n)$ are $\left(n, r, c_{2}\right)=$ $(n,|T|, n /|T|)$.

So far all observations were easy consequences from a combination of known facts about antipodal regular covers of complete graphs. In order really to obtain new such covers, in the following theorem we describe a method for obtaining a skew $\operatorname{gH}\left(T, n^{2}\right)$ from any $\mathrm{gH}(T, n)$.

Theorem 5.6. Let $T$ be a finite abelian group and let $H=\left(h_{i, j}\right)$ be any $\mathrm{gH}(T, n)$. Let $\psi$ : $\{1,2, \ldots, n\}^{2} \rightarrow\left\{1,2, \ldots, n^{2}\right\}$ be any bijection. Define the matrix $R_{H}=\left(r_{(i, j)^{\psi},(k, l)^{\psi}}\right)$ of order $n^{2}$ over $T$ according to

$$
r_{(i, j) \psi,(k, l) \psi}:=h_{k, j} \cdot h_{i, l}^{-1}
$$

Then $R_{H}$ is a skew $\mathrm{gH}\left(T, n^{2}\right)$.

Proof. Part 1. The matrix $R_{H}$ is self-adjoint: Let $(i, j),(k, l) \in\{1, \ldots, n\}^{2}$. Observe that

$$
r_{(k, l)^{\psi},(i, j)^{\psi}}=h_{i, l} h_{k, j}^{-1}=\left(h_{k, j} \cdot h_{i, l}^{-1}\right)^{-1}=\left(r_{(i, j)^{\psi},(k, l) \psi}\right)^{-1} .
$$

Part 2. The matrix $R_{H}$ has on diagonal everywhere the element $\underline{e}$ : Indeed, for $(i, j) \in$ $\{1, \ldots, n\}^{2}$, observe that

$$
r_{(i, j)^{\psi},(i, j) \psi}=h_{i, j} h_{i, j}^{-1}=\underline{e} .
$$

Part 3. Let us show that $R_{H}$ is a generalized Hadamard matrix: Let $\left(i_{1}, j_{1}\right),\left(i_{2}, j_{2}\right)$ be two distinct elements of $\{1, \ldots, n\}^{2}$. Then

$$
\begin{aligned}
\sum_{(k, l) \in\{1, \ldots, n\}^{2}} r_{\left(i_{1}, j_{1}\right)^{\psi},(k, l) \psi} r_{\left(i_{2}, j_{2}\right)^{\psi},(k, l) \psi}^{-1} & =\sum_{(k, l) \in\{1, \ldots, n\}^{2}}\left(h_{k, j_{1}} h_{i_{1}, l}^{-1}\right)\left(h_{k, j_{2}} h_{i_{2}, l}^{-1}\right)^{-1} \\
& =\sum_{(k, l) \in\{1, \ldots, n\}^{2}}\left(h_{k, j_{1}} h_{i_{1}, l}^{-1}\right)\left(h_{i_{2}, l} h_{k, j_{2}}^{-1}\right) \\
& =\sum_{k=1}^{n} h_{k, j_{1}}\left(\sum_{l=1}^{n} h_{i_{1}, l^{-1}}^{-1} h_{i_{2}, l}\right) h_{k, j_{2}}^{-1} .
\end{aligned}
$$


if $i_{1}=i_{2}$, then $j_{1} \neq j_{2}$, and we compute

$$
\begin{aligned}
\sum_{k=1}^{n} h_{k, j_{1}}\left(\sum_{l=1}^{n} h_{i_{1}, l}^{-1} h_{i_{2}, l}\right) h_{k, j_{2}}^{-1} & =\sum_{k=1}^{n} h_{k, j_{1}}(n \cdot \underline{e}) h_{k, j_{2}}^{-1} \\
& =n \cdot \underline{e} \cdot \sum_{k=1}^{n} h_{k, j_{1}} h_{k, j_{2}}^{-1} \\
& =\frac{n^{2}}{|T|} \underline{T} .
\end{aligned}
$$

If $i_{1} \neq i_{2}$, then, using that $T$ is abelian, we obtain

$$
\begin{aligned}
\sum_{k=1}^{n} h_{k, j_{1}}\left(\sum_{l=1}^{n} h_{i_{1}, l}^{-1} h_{i_{2}, l}\right) h_{k, j_{2}}^{-1} & =\sum_{k=1}^{n} h_{k, j_{1}}\left(\frac{n}{|T|} \cdot \underline{T}\right) h_{k, j_{2}}^{-1} \\
& =\frac{n^{2}}{|T|} \underline{T} .
\end{aligned}
$$

Remark 5.7. The condition in Theorem 5.6 that $T$ be abelian can be dropped if instead we assume that also $H^{\top}$ is a generalized Hadamard matrix, too. This is equivalent to the assumption that the element wise conjugation $H^{-}$of $H$ is a generalized Hadamard matrix. However, we are not aware of any such matrices, except when $T$ is abelian. In this case both conditions are always fulfilled.

Corollary 5.8. For a finite abelian group $T$, if there is a $\mathrm{gH}(T, n)$, then for all $t \in \mathbb{N} \backslash\{0\}$ there is a skew $\mathrm{gH}\left(T, n^{2^{t}}\right)$.

Remark 5.9. In the construction of $R_{H}$ from $H$ the choice of the bijection $\psi$ is not of importance, because different choices will give equivalent skew generalized Hadamard matrices that give rise to isomorphic covers of $K_{n}$. So further on it will be convenient to assume that in the construction $\psi$ is taken such that $(i, j)^{\psi}=(i-1) n+j$ where $n$ is the order of $H$.

Before exploring several interesting examples that we obtain from Theorem 5.6, let us give an alternative description of the construction using block matrices:

As before, let $H$ be a generalized Hadamard matrix $\mathrm{gH}(T, n)$ such that $H^{-}$is a generalized Hadamard matrix, too. Take $\varphi:\{1,2, \ldots, n\}^{2} \rightarrow\left\{1,2, \ldots, n^{2}\right\}$ defined by $(k, l) \mapsto(l-1) n+k$. Moreover, take $\psi$ as defined previously. Now define the matrix $R_{H}^{\prime}=\left(r_{\left.(i, j)^{\psi},(k, l)^{\varphi}\right)}\right.$ of order $n^{2}$. A close inspection reveals that the thus defined matrix is of block-shape $R_{H}^{\prime}=H^{\top} \otimes H^{-}$. More concretely

$$
R_{H}^{\prime}=\left(\begin{array}{cccc}
H^{\top} \cdot h_{1,1}^{-1} & H^{\top} \cdot h_{2,1}^{-1} & \ldots & H^{\top} \cdot h_{n, 1}^{-1} \\
H^{\top} \cdot h_{1,2}^{-1} & H^{\top} \cdot h_{2,2}^{-1} & \ldots & H^{\top} \cdot h_{n, 2}^{-1} \\
\vdots & \vdots & \ddots & \vdots \\
H^{\top} \cdot h_{1, n}^{-1} & H^{\top} \cdot h_{2, n}^{-1} & \ldots & H^{\top} \cdot h_{n, n}^{-1}
\end{array}\right)
$$

If we permute the columns of $R_{H}^{\prime}$ by the permutation $\varphi^{-1} \psi$, then we obtain $R_{H}$. 


\section{Small examples}

In this section we will survey small generalized Hadamard matrices and the covers obtained from them using the construction described above. In Table 2 we collect the parameters of small generalized Hadamard matrices (of order $\leq 15$ ) and of the regular covers constructed from them ${ }^{5}$. In [40] all generalized Hadamard matrices of order $\leq 15$ are enumerated.

\begin{tabular}{c|l|l|c|c}
\hline$\#$ & parameters of $H$ & parameters of $R_{H}$ & $\left(n, r, c_{2}\right)$ of $\Gamma^{R_{H}}$ & $\left|V\left(\Gamma^{R_{H}}\right)\right|$ \\
\hline 1 & $\mathrm{gH}\left(E_{2}, 2\right)$ & $\mathrm{gH}\left(E_{2}, 4\right)$ & $(4,2,2)$ & 8 \\
\hline 2 & $\mathrm{gH}\left(E_{3}, 3\right)$ & $\mathrm{gH}\left(E_{3}, 9\right)$ & $(9,3,3)$ & 27 \\
\hline 3 & $\mathrm{gH}\left(E_{4}, 4\right)$ & $\mathrm{gH}\left(E_{4}, 16\right)$ & $(16,4,4)$ & 64 \\
\hline 4 & $\mathrm{gH}\left(E_{2}, 4\right)$ & $\mathrm{gH}\left(E_{2}, 16\right)$ & $(16,2,8)$ & 32 \\
\hline 5 & $\mathrm{gH}\left(E_{5}, 5\right)$ & $\mathrm{gH}\left(E_{5}, 25\right)$ & $(25,5,5)$ & 125 \\
\hline $\mathbf{6}$ & $\mathrm{gH}\left(\mathbf{E}_{\mathbf{3}}, \mathbf{6}\right)$ & $\mathrm{gH}\left(\mathbf{E}_{\mathbf{3}}, \mathbf{3 6}\right)$ & $(\mathbf{3 6}, \mathbf{3}, \mathbf{1 2})$ & $\mathbf{1 0 8}$ \\
\hline 7 & $\mathrm{gH}\left(E_{7}, 7\right)$ & $\mathrm{gH}\left(E_{7}, 49\right)$ & $(49,7,7)$ & 343 \\
\hline 8 & $\mathrm{gH}\left(E_{8}, 8\right)$ & $\mathrm{gH}\left(E_{8}, 64\right)$ & $(64,8,8)$ & 512 \\
\hline 9 & $\mathrm{gH}\left(E_{4}, 8\right)$ & $\mathrm{gH}\left(E_{4}, 64\right)$ & $(64,4,16)$ & 256 \\
\hline 10 & $\mathrm{gH}\left(E_{2}, 8\right)$ & $\mathrm{gH}\left(E_{2}, 64\right)$ & $(64,2,32)$ & 128 \\
\hline $\mathbf{1 1}$ & $\mathrm{gH}\left(\mathbf{E}_{\mathbf{9}}, \mathbf{9}\right)$ & $\mathrm{gH}\left(\mathbf{E}_{\mathbf{9}}, \mathbf{8 1}\right)$ & $(\mathbf{8 1}, \mathbf{9}, \mathbf{9})$ & $\mathbf{7 2 9}$ \\
\hline $\mathbf{1 2}$ & $\mathrm{gH}\left(\mathbf{E}_{\mathbf{3}}, \mathbf{9}\right)$ & $\mathrm{gH}\left(\mathbf{E}_{\mathbf{3}}, \mathbf{8 1}\right)$ & $(\mathbf{8 1}, \mathbf{3}, \mathbf{2 7})$ & $\mathbf{2 4 3}$ \\
\hline $\mathbf{1 3}$ & $\mathrm{gH}\left(\mathbf{E}_{\mathbf{5}}, \mathbf{1 0}\right)$ & $\mathrm{gH}\left(\mathbf{E}_{\mathbf{5}}, \mathbf{1 0 0}\right)$ & $(\mathbf{1 0 0}, \mathbf{5}, \mathbf{2 0})$ & $\mathbf{5 0 0}$ \\
\hline 14 & $\mathrm{gH}\left(E_{11}, 11\right)$ & $\mathrm{gH}\left(E_{11}, 121\right)$ & $(121,11,11)$ & 1331 \\
\hline $\mathbf{1 5}$ & $\mathrm{gH}\left(\mathbf{E}_{\mathbf{3}}, \mathbf{1 2}\right)$ & $\mathrm{gH}\left(\mathbf{E}_{\mathbf{3}}, \mathbf{1 4 4}\right)$ & $(\mathbf{1 4 4}, \mathbf{3}, \mathbf{4 8})$ & $\mathbf{4 3 2}$ \\
\hline $\mathbf{1 6}$ & $\mathrm{gH}\left(\mathbf{E}_{\mathbf{4}}, \mathbf{1 2}\right)$ & $\mathrm{gH}\left(\mathbf{E}_{\mathbf{4}}, \mathbf{1 4 4}\right)$ & $(\mathbf{1 4 4}, \mathbf{4}, \mathbf{3 6})$ & $\mathbf{5 7 6}$ \\
\hline $\mathbf{1 7}$ & $\mathrm{gH}\left(\mathbf{E}_{\mathbf{2}}, \mathbf{1 2}\right)$ & $\mathrm{gH}\left(\mathbf{E}_{\mathbf{2}}, \mathbf{1 4 4}\right)$ & $(\mathbf{1 4 4}, \mathbf{2}, \mathbf{7 2})$ & $\mathbf{2 8 8}$ \\
\hline 18 & $\mathrm{gH}\left(E_{13}, 13\right)$ & $\mathrm{gH}\left(E_{13}, 169\right)$ & $(169,13,13)$ & 2197 \\
\hline $\mathbf{1 9}$ & $\mathrm{gH}\left(\mathbf{E}_{\mathbf{7}}, \mathbf{1 4}\right)$ & $\mathrm{gH}\left(\mathbf{E}_{\mathbf{7}}, \mathbf{1 9 6}\right)$ & $(\mathbf{1 9 6}, \mathbf{7}, \mathbf{2 8})$ & $\mathbf{1 3 7 2}$ \\
\hline & & & &
\end{tabular}

Table 2: Small regular covers with $\delta=-2$ obtained from Theorem 5.6.

From each such generalized Hadamard matrix we computed the associated regular cover. Generalized Hadamard matrices of type $\mathrm{gH}\left(E_{q}, q\right)$ correspond to certain projective planes. Those that correspond to Desarguesian planes can be obtained as multiplication tables of $G F(q)$. They generally lead to distance transitive graphs (recall that all such graphs are classified in [46]). Most of the known generalized Hadamard matrices $\mathrm{gH}\left(E_{d}, q\right)$ for $d \mid q$ can be obtained as homomorphic images of $\mathrm{gH}\left(E_{q}, q\right)$. This is the case which appears in many rows of Table 2 . Of a special interest are lines \#6, \#11, \#12, \#13, \#15, \#16, \#17, \#19 since in these cases there exist generalized Hadamard matrices that lead to regular covers that are not distance transitive. Therefore to each of these lines we will dedicate a special subsection with explanations of their appearance.

\footnotetext{
${ }^{5}$ Note that, according to [40], there are no generalized Hadamard matrices of order equal to 15
} 


\subsection{About \#6}

There is, up to monomial equivalence, just one $\mathrm{gH}\left(\mathbb{Z}_{3}, 6\right)$ :

$$
H_{6}=\left(\begin{array}{cccccc}
0 & 0 & 0 & 0 & 0 & 0 \\
0 & 0 & 1 & -1 & -1 & 1 \\
0 & 1 & 0 & 1 & -1 & -1 \\
0 & -1 & 1 & 0 & 1 & -1 \\
0 & -1 & -1 & 1 & 0 & 1 \\
0 & 1 & -1 & -1 & 1 & 0
\end{array}\right)
$$

This is in a sense the smallest "interesting" generalized Hadamard matrix, insofar as it can not be obtained as a homomorphic image of the multiplication table of a finite field. The construction given in Theorem 5.6 together with $\psi:(i, j) \mapsto 6(i-1)+j$ gives the following skew $\mathrm{gH}\left(\mathbb{Z}_{3}, 36\right)$ :

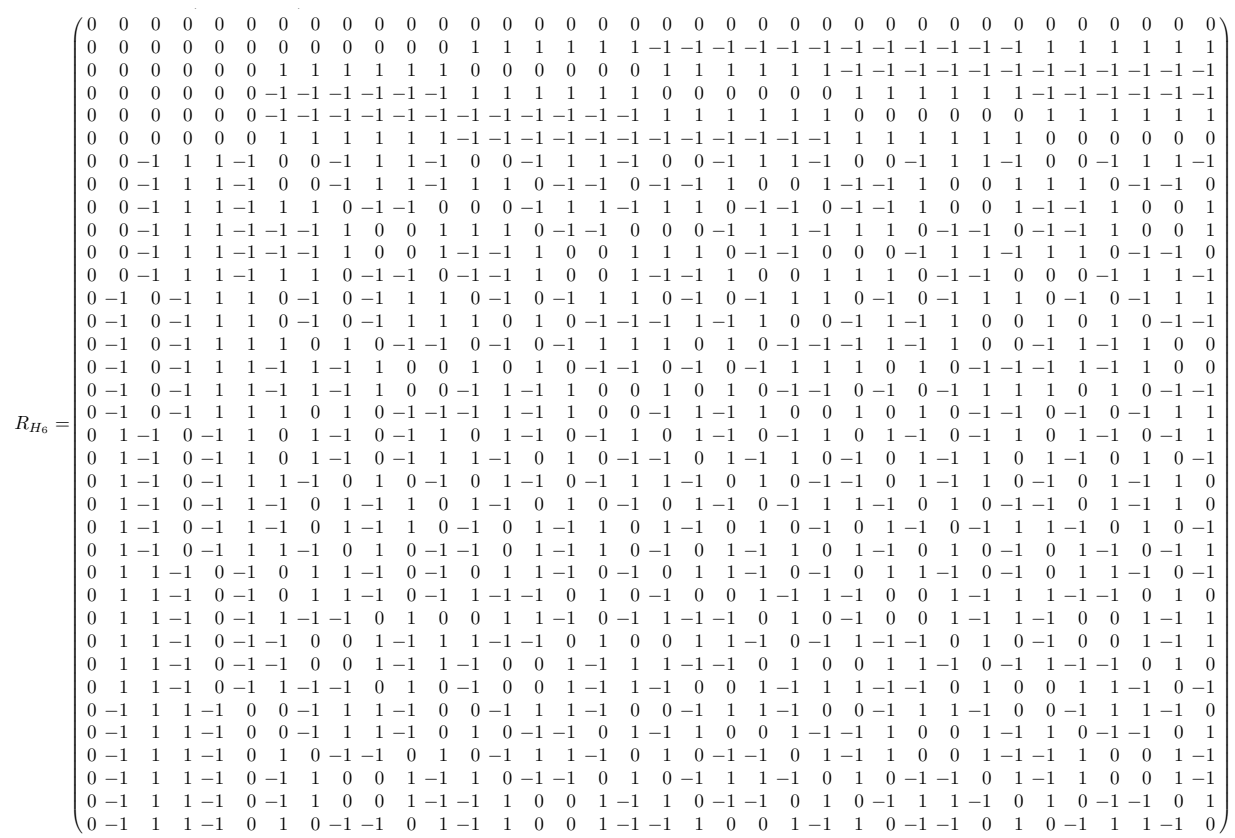

In turn, the matrix $R_{H_{6}}$ defines a regular cover of $K_{36}$ with parameters $\left(n, r, c_{2}\right)=(36,3$, 12 ) which is not distance transitive. Its automorphism group is isomorphic to $\left(\mathbb{Z}_{3} \cdot S_{6}\right) \cdot \mathbb{Z}_{2}$. This group acts transitively with rank 8 on the 108 vertices of the cover.

The matrix $H_{6}$ defines a class-regular $\operatorname{RTD}_{2}(6,3)$. In fact, this resolvable transversal design is at the same time an elation semibiplane $\operatorname{SBP}(18,6)$. This semibiplane was announced in [62], see the extra discussion in Section 10.2.

\subsection{About \#11}

There exist, up to monomial equivalence, two generalized Hadamard matrices $\operatorname{gH}\left(E_{9}, 9\right)$. One of them is the multiplication table of GF(9). It corresponds to the Desarguesian projective plane, and it leads to a distance transitive $(81,9,9)$-cover. The second one is the 
multiplication table of the Dickson near field with parameters $(3,2)$ (cf. [85, Chap. 8]). It corresponds to the Hall plane and it gives rise to a non distance transitive regular cover with parameters $(81,9,9)$. Its automorphism group coincides with the automorphism group of the incidence graph of the corresponding (non self-dual) $\operatorname{RTD}_{1}(9,9)$. It has order 31104 and acts on vertices of the cover with rank 39. Moreover, it has two orbits on vertices, one of length 81 , and one of length 648 . GAP identified its structure as $\left(\left(\mathbb{Z}_{3}^{2}: Q_{8}\right)^{2}: \mathbb{Z}_{3}\right): \mathbb{Z}_{2}$.

\subsection{About \#12}

In [77], as a byproduct, all $\mathrm{gH}\left(\mathbb{Z}_{3}, 9\right)$ are characterized up to monomial equivalence. It turns out that there are two such generalized Hadamard matrices. One of them is obtained by projecting the multiplication table of $\mathrm{GF}(9)$ onto $\mathbb{Z}_{3}$. The Godsil-Hensel matrix constructed from it, using Theorem 5.6, yields a distance transitive regular $(81,3,27)$-cover. However, the second example is of a greater interest in the current context since it gives rise to a regular cover that is not distance transitive. We extracted the corresponding $\mathrm{gH}\left(\mathbb{Z}_{3}, 9\right)$ from [77] (note that this matrix was first constructed in [6]):

$$
H_{12}=\left(\begin{array}{ccccccccc}
0 & 0 & 0 & 0 & 0 & 0 & 0 & 0 & 0 \\
0 & 0 & 1 & 0 & -1 & -1 & 1 & 1 & -1 \\
0 & 0 & 0 & 1 & 1 & 1 & -1 & -1 & -1 \\
0 & 1 & 0 & -1 & -1 & 0 & -1 & 1 & 1 \\
0 & -1 & -1 & 1 & -1 & 1 & 0 & 1 & 0 \\
0 & 1 & -1 & 0 & 1 & -1 & 0 & -1 & 1 \\
0 & 1 & -1 & -1 & 0 & 1 & 1 & 0 & -1 \\
0 & -1 & 1 & -1 & 1 & 0 & 1 & -1 & 0 \\
0 & -1 & 1 & 1 & 0 & -1 & -1 & 0 & 1
\end{array}\right)
$$

The class regular $\operatorname{RTD}_{3}(9,3)$ that corresponds to this $\mathrm{gH}\left(\mathbb{Z}_{3}, 9\right)$ has an automorphism group of order 2916 that acts point- and block-transitively. The incidence graph has an automorphism group of order 5832. Thus the design is self-dual. In contrast to all other examples given here and below, the regular $(81,3,27)$-cover that arises from this generalized Hadamard matrix has essentially more symmetries. Its automorphism group has order 52488 . This group acts transitively of rank 12 on the vertices of the cover. See Section 10.6 for further discussion.

\subsection{About \#13}

In [62], except for the $\operatorname{SBP}(18,6)$ that was considered above, there was also given an example of an elation semibiplane $\operatorname{SBP}(50,10)$. A bit of creative reading of the construction reveals that this semibiplane can be constructed in a way analogous to the previous one, starting from the following $\mathrm{gH}\left(\mathbb{Z}_{5}, 10\right)$ : 


$$
H_{13}=\left(\begin{array}{cccccccccc}
0 & 0 & 0 & 0 & 0 & 0 & 0 & 0 & 0 & 0 \\
0 & 0 & 1 & 1 & 2 & 2 & -2 & -2 & -1 & -1 \\
0 & 1 & 1 & -2 & 0 & -1 & 2 & -1 & 2 & -2 \\
0 & 1 & -2 & 1 & -1 & 0 & -1 & 2 & -2 & 2 \\
0 & 2 & 0 & -1 & -2 & 2 & -1 & 1 & 1 & -2 \\
0 & 2 & -1 & 0 & 2 & -2 & 1 & -1 & -2 & 1 \\
0 & -2 & 2 & -1 & -1 & 1 & 2 & -2 & 0 & 1 \\
0 & -2 & -1 & 2 & 1 & -1 & -2 & 2 & 1 & 0 \\
0 & -1 & 2 & -2 & 1 & -2 & 0 & 1 & -1 & 2 \\
0 & -1 & -2 & 2 & -2 & 1 & 1 & 0 & 2 & -1
\end{array}\right)
$$

As was mentioned above, this semibiplane is at the same time a residual transversal design $\mathrm{RTD}_{2}(10,5)$ and its incidence graph is a bipartite, antipodal distance regular graph with parameters $(10,9,8,1 ; 1,2,9,10)$.

The automorphism group of the $\operatorname{RTD}_{2}(10,5)$ has order 2000 . It acts transitively on vertices and it has two orbits, each of length 250 , on edges (that is flags of $\operatorname{RTD}_{2}(10,5)$ ). The stabilizer of a vertex is isomorphic to $\operatorname{AGL}(1,5)$, the stabilizer of an edge from one orbit is isomorphic to $\mathbb{Z}_{4} \times \mathbb{Z}_{2}$ and the stabilizer of an edge from the second orbit is isomorphic to $\mathbb{Z}_{8}$. The group is isomorphic to the automorphism group of the related regular cover, see again Section 10.4 for further discussion.

\subsection{A common generalization of \#6 and \#13}

It turns out that the generalized Hadamard matrices $\mathrm{gH}\left(\mathbb{Z}_{3}, 6\right)$ and $\mathrm{gH}\left(\mathbb{Z}_{5}, 10\right)$ corresponding to rows \#6 and \#13 are the first two elements in an infinite series.

In [4, Thm. VIII.3.14] there is given a construction of generalized Hadamard matrices $\mathrm{gH}\left(E_{q}, 2 q\right)$ for arbitrary prime powers $q$. The $\mathrm{gH}\left(\mathbb{Z}_{3}, 6\right)$ and $\mathrm{gH}\left(\mathbb{Z}_{5}, 10\right)$ given above are monomially equivalent to the cases $q=3$ and $q=5$, respectively. The construction is attributed to [64] and [103]. However, the part that is of interest here, namely when $q$ is a prime, was handled much earlier in [75] and [13].

Each of the $\mathrm{gH}\left(E_{q}, 2 q\right)$ gives rise to a class-regular $\operatorname{RTD}_{2}(2 q, q)$ that is, an elation semibiplane $\operatorname{SBP}\left(2 q^{2}, 2 q\right)$.

\subsection{About \#15}

The following $\mathrm{gH}\left(\mathbb{Z}_{3}, 12\right)$ was constructed "manually" by E.A. Seiden in [93].

$$
H_{15}=\left(\begin{array}{rrrrrrrrrrrr}
0 & 0 & 0 & 0 & 0 & 0 & 0 & 0 & 0 & 0 & 0 & 0 \\
0 & 0 & 0 & 0 & 1 & 1 & 1 & 1 & -1 & -1 & -1 & -1 \\
0 & 0 & 1 & -1 & -1 & -1 & 0 & 1 & 1 & 1 & -1 & 0 \\
1 & 0 & 0 & -1 & -1 & 1 & 0 & -1 & -1 & 0 & 1 & 1 \\
1 & -1 & 0 & 0 & 0 & -1 & -1 & 1 & 1 & 0 & -1 & 1 \\
0 & 0 & -1 & 1 & 0 & 1 & -1 & -1 & 0 & 1 & -1 & 1 \\
0 & -1 & 1 & 0 & 1 & -1 & 0 & -1 & 0 & -1 & 1 & 1 \\
1 & 0 & -1 & 0 & 1 & -1 & -1 & 0 & -1 & 1 & 1 & 0 \\
0 & -1 & 0 & 1 & -1 & -1 & 1 & 0 & -1 & 1 & 0 & 1 \\
-1 & 0 & 0 & 1 & 0 & -1 & 1 & -1 & 1 & -1 & 1 & 0 \\
-1 & 0 & 1 & 0 & -1 & 1 & -1 & 0 & 1 & -1 & 0 & 1 \\
0 & 1 & 0 & 0 & -1 & 0 & -1 & -1 & 1 & 1 & 1 & -1
\end{array}\right)
$$


The automorphism group of the associated regular cover has order 1728. It has two orbits, each of size 216 on the 432 vertices of the cover. GAP identified it as $\left(\left(\left(\mathbb{Z}_{2} \times \mathbb{Z}_{6} \times A_{4}\right)\right.\right.$ : $\left.\left.\mathbb{Z}_{3}\right): \mathbb{Z}_{2}\right): \mathbb{Z}_{2}$.

\subsection{About \#16}

The following is a $\mathrm{gH}\left(E_{4}, 12\right)$ :

$$
H_{16}=\left(\begin{array}{cccccccccccc}
0 & 0 & 0 & 0 & 0 & 0 & 0 & 0 & 0 & 0 & 0 & 0 \\
0 & 0 & 0 & b & b & b & c & c & c & a & a & a \\
0 & 0 & 0 & c & c & c & a & a & a & b & b & b \\
0 & c & b & a & b & c & b & a & 0 & c & 0 & a \\
0 & c & b & c & a & b & 0 & b & a & a & c & 0 \\
0 & c & b & b & c & a & a & 0 & b & 0 & a & c \\
0 & b & a & c & 0 & a & b & 0 & c & b & c & a \\
0 & b & a & a & c & 0 & c & b & 0 & a & b & c \\
0 & b & a & 0 & a & c & 0 & c & b & c & a & b \\
0 & a & c & b & a & 0 & b & c & a & b & 0 & c \\
0 & a & c & 0 & b & a & a & b & c & c & b & 0 \\
0 & a & c & a & 0 & b & c & a & b & 0 & c & b
\end{array}\right),
$$

where $E_{4}=\{0, a, b, c\}$, and where $a+b=c$. It is taken from [4, Ex. VIII.3.19], but it was discovered by J. Seberry in [91]. The automorphism group of the related regular cover has order 648 . GAP identified the structure of this group as $\left(\left(\left(\mathbb{Z}_{3} \times \mathbb{Z}_{3}\right): \mathbb{Z}_{3}\right): \mathbb{Z}_{2}\right): A_{4}$.

\subsection{About \#17}

Up to equivalence, there is a unique generalized Hadamard matrix $\mathrm{gH}\left(\mathbb{Z}_{2}, 12\right)$ (this is of course also a usual Hadamard matrix). One way to obtain it is to start with the $\operatorname{gH}\left(E_{4}, 12\right)$ given above and to take its image under the epimorphism $\varphi: E_{4} \rightarrow \mathbb{Z}_{2}$ with $\varphi(b)=$ $\varphi(c)=1$, and $\varphi(a)=\varphi(0)=0$. Another, more standard, way relies on the observation that this matrix is equivalent to the Paley-Hadamard matrix $P_{11}$ of order 12 (in the sense of [83]). The corresponding $\operatorname{RTD}_{6}(12,2)$ has automorphism group $2 . M_{12}$ and its incidence graph has automorphism group $\left(\mathbb{Z}_{2} \cdot M_{12}\right): \mathbb{Z}_{2}$. However, the automorphism group of the corresponding regular double cover of $K_{144}$ is isomorphic to $\mathbb{Z}_{2} \times\left(M_{12}: \mathbb{Z}_{2}\right)$. We refer to Section 10.5 for a discussion of this famous example. 


\subsection{About \#19}

The unique $\mathrm{gH}\left(E_{7}, 14\right)$ is the third member of the infinite series of generalized Hadamard matrices mentioned in Section 6.5:

$$
H_{19}=\left(\begin{array}{rrrrrrrrrrrrrr}
0 & 0 & 0 & 0 & 0 & 0 & 0 & 0 & 0 & 0 & 0 & 0 & 0 & 0 \\
2 & 3 & -3 & -2 & -1 & 0 & 1 & -1 & 0 & 1 & 2 & 3 & -3 & -2 \\
1 & 3 & -2 & 0 & 2 & -3 & -1 & 3 & -2 & 0 & 2 & -3 & -1 & 1 \\
-3 & 0 & 3 & -1 & 2 & -2 & 1 & -2 & 1 & -3 & 0 & 3 & -1 & 2 \\
-3 & 1 & -2 & 2 & -1 & 3 & 0 & -2 & 2 & -1 & 3 & 0 & -3 & 1 \\
1 & -1 & -3 & 2 & 0 & -2 & 3 & 3 & 1 & -1 & -3 & 2 & 0 & -2 \\
2 & 1 & 0 & -1 & -2 & -3 & 3 & -1 & -2 & -3 & 3 & 2 & 1 & 0 \\
0 & -1 & 3 & -2 & -2 & 3 & -1 & 0 & 2 & 1 & -3 & -3 & 1 & 2 \\
-2 & -2 & 3 & -1 & 0 & -1 & 3 & -3 & -3 & 1 & 2 & 0 & 2 & 1 \\
-1 & 0 & -1 & 3 & -2 & -2 & 3 & 2 & 0 & 2 & 1 & -3 & -3 & 1 \\
3 & -2 & -2 & 3 & -1 & 0 & -1 & 1 & -3 & -3 & 1 & 2 & 0 & 2 \\
3 & -1 & 0 & -1 & 3 & -2 & -2 & 1 & 2 & 0 & 2 & 1 & -3 & -3 \\
-1 & 3 & -2 & -2 & 3 & -1 & 0 & 2 & 1 & -3 & -3 & 1 & 2 & 0 \\
-2 & 3 & -1 & 0 & -1 & 3 & -2 & -3 & 1 & 2 & 0 & 2 & 1 & -3
\end{array}\right)
$$

The related regular $(196,7,28)$-cover has an automorphism group of order 8232 which acts intransitively and of rank 242 on the vertices of the cover. The two orbits on vertices both have length 686 . The group is isomorphic to the automorphism group of the incidence graph of the related (self-dual) $\mathrm{RTD}_{2}(14,7)$. GAP identified the structure of the group as $\left(\left(\left(\mathbb{Z}_{7} \times \mathbb{Z}_{7}\right): \mathbb{Z}_{7}\right): Q_{8}\right): \mathbb{Z}_{3}$.

\section{Towards a more general construction}

In the previous section (as a byproduct of the developed techniques) it was described how to obtain a regular cover of $K_{n^{2}}$ from any regular distance regular cover of $K_{n, n}$ (i.e. from any generalized Hadamard matrix). In this section we demonstrate a possible generalization of these techniques. This means that instead of starting from an antipodal regular cover of a complete bipartite graph (i.e. from a generalized Hadamard matrix), the antipodal cover of a suitable bipartite DRG will be exploited. Namely, starting from the Foster graph (the unique 3-fold regular cover of the collinearity graph of the $\operatorname{GQ}(2,2)=W_{2}$ ) a 3fold distance regular cover of $K_{45}$ will be constructed. The construction of its GodsilHensel matrix will be quite similar to the one given in Theorem 5.6. This was one of the motivations to present this result in this paper together with the construction that uses generalized Hadamard matrices.

\section{1 $X \times Y$-matrices over group rings}

For a better understanding of the construction a more flexible definition of matrices is suggested: Let $X$ and $Y$ be finite sets, let $T$ be a finite group, and let $\mathbb{Z}(T)$ be the group ring of $G$. An $X \times Y$-matrix over $\mathbb{Z}(T)$ is just a function from $X \times Y$ to $\mathbb{Z}(T)$. As usual, an $X \times Y$-matrix $A$ can be denoted conveniently as $(X \times Y)$-indexed family $\left(a_{x, y}\right)_{x \in X, y \in Y}$ where $a_{x, y}=A(x, y)$. The sum of $X \times Y$-matrices is defined as usual, pointwise. For an $X \times Y$-matrix $A=\left(a_{x, y}\right)$ and a $Y \times Z$-matrix $B=\left(b_{y, z}\right)$, the product $A B$ is the 
$X \times Z$-matrix $\left(c_{x, z}\right)$ where

$$
c_{x, z}=\sum_{y \in Y} a_{x, y} \cdot b_{y, z} .
$$

Note that the matrices of order $n$ that were defined in Section 4.1 are special cases of $X \times Y$-matrices for $X=Y=\{1,2, \ldots, n\}$.

\subsection{The unique generalized quadrangle $\mathrm{GQ}(2,2)$}

Recall the construction of the unique $\operatorname{GQ}(2,2)$, also known as $W_{2}$ (see Figure 1, below). Its points can be taken as the 2-element subsets of $\{1,2, \ldots, 6\}$ and its lines are (unordered) triples of distinct mutually disjoint points. The incidence relation is the usual containment relation. The incidence structure is given in Table 3, while the famous picture, depicting $W_{2}$ (due to S. Payne) is presented in Figure 1. The incidence graph of $W_{2}$ has as vertices

\begin{tabular}{|c||c|c|c|c|c|c|c|c|c|c|c|c|c|c|c|}
\hline & 12 & 14 & 13 & 16 & 15 & 12 & 12 & 14 & 14 & 16 & 15 & 16 & 15 & 13 & 13 \\
& 34 & 23 & 24 & 25 & 26 & 36 & 35 & 26 & 25 & 23 & 23 & 24 & 24 & 26 & 25 \\
& 56 & 56 & 56 & 34 & 34 & 45 & 46 & 35 & 36 & 45 & 46 & 35 & 36 & 45 & 46 \\
\hline 56 & $\times$ & $\times$ & $\times$ & & & & & & & & & & & & \\
\hline 34 & $\times$ & & & $\times$ & $\times$ & & & & & & & & & & \\
\hline 12 & $\times$ & & & & & $\times$ & $\times$ & & & & & & & & \\
\hline 14 & & $\times$ & & & & & & $\times$ & $\times$ & & & & & & \\
\hline 23 & & $\times$ & & & & & & & & $\times$ & $\times$ & & & & \\
\hline 24 & & & $\times$ & & & & & & & & & $\times$ & $\times$ & & \\
\hline 13 & & & $\times$ & & & & & & & & & & & $\times$ & $\times$ \\
\hline 25 & & & & $\times$ & & & & & $\times$ & & & & & & $\times$ \\
\hline 16 & & & & $\times$ & & & & & & $\times$ & & $\times$ & & & \\
\hline 15 & & & & & $\times$ & & & & & & $\times$ & & $\times$ & & \\
\hline 26 & & & & & $\times$ & & & $\times$ & & & & & & $\times$ & \\
\hline 36 & & & & & & $\times$ & & & $\times$ & & & & $\times$ & & \\
\hline 45 & & & & & & $\times$ & & & & $\times$ & & & & $\times$ & \\
\hline 46 & & & & & & & $\times$ & & & & $\times$ & & & & $\times$ \\
\hline 35 & & & & & & & $\times$ & $\times$ & & & & $\times$ & & & \\
\hline
\end{tabular}

Table 3: The incidence table of $W_{2}$.

the points and lines of $W_{2}$, and the adjacency relation is the symmetrized incidence relation. The graph shall be denoted by $L\left(W_{2}\right)$. It is the unique distance regular graph with parameters $(3,2,2,2 ; 1,1,1,3)$. It is also known under the name "Tutte's 8-cage".

\subsection{The Foster graph}

This is the unique distance regular graph with parameters

$$
(3,2,2,2,2,1,1,1 ; 1,1,1,1,2,2,2,3) .
$$

It is antipodal and bipartite. It can be obtained as the incidence graph of a regular three-fold cover $\widetilde{W}_{2}$ of $W_{2}$. The collapsed weighted incidence matrix of this cover is given in Table 4 . 


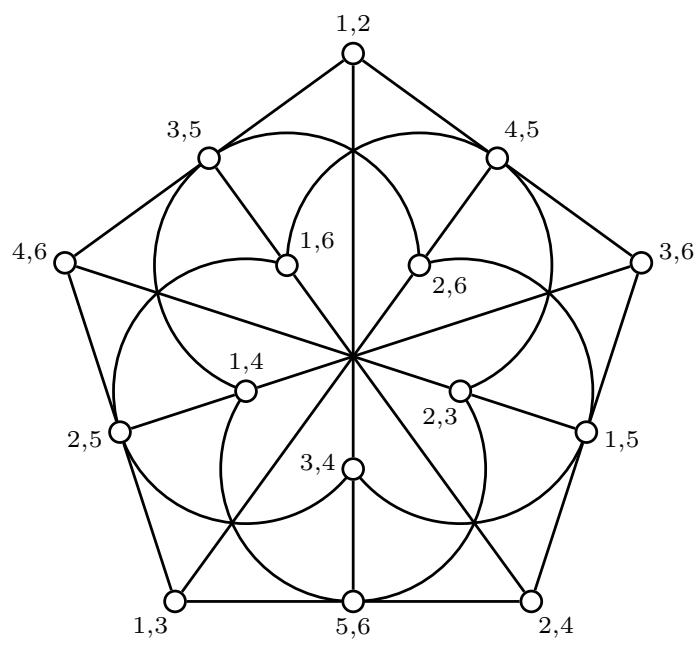

Figure 1: $W_{2}$, aka Payne's doily.

Each antipodal fibre has size 3 . The entries of this matrix are elements of $\mathbb{Z}_{3}$. If these are replaced by permutation matrices of the regular representation of $\mathbb{Z}_{3}$, then a 3 -fold regular cover of $W_{2}$ is obtained.

\begin{tabular}{|l||c|c|c|c|c|c|c|c|c|c|c|c|c|c|c|}
\hline & 12 & 14 & 13 & 16 & 15 & 12 & 12 & 14 & 14 & 16 & 15 & 16 & 15 & 13 & 13 \\
& 34 & 23 & 24 & 25 & 26 & 36 & 35 & 26 & 25 & 23 & 23 & 24 & 24 & 26 & 25 \\
& 56 & 56 & 56 & 34 & 34 & 45 & 46 & 35 & 36 & 45 & 46 & 35 & 36 & 45 & 46 \\
\hline \hline 56 & 0 & 0 & 0 & & & & & & & & & & & & \\
\hline 34 & 0 & & & 0 & 0 & & & & & & & & & & \\
\hline 12 & 0 & & & & & 0 & 0 & & & & & & & & \\
\hline 14 & & 0 & & & & & & 0 & 0 & & & & & & \\
\hline 23 & & 0 & & & & & & & & 0 & 0 & & & & \\
\hline 24 & & & 0 & & & & & & & & & 0 & 0 & & \\
\hline 13 & & & 0 & & & & & & & & & & & 0 & 0 \\
\hline 25 & & & & 0 & & & & & 1 & & & & & & -1 \\
\hline 16 & & & & 0 & & & & & & -1 & & 1 & & & \\
\hline 15 & & & & & 0 & & & & & & 1 & & -1 & & \\
\hline 26 & & & & & 0 & & & -1 & & & & & & 1 & \\
\hline 36 & & & & & & 0 & & & -1 & & & & 1 & & \\
\hline 45 & & & & & & 0 & & & & 1 & & & & -1 & \\
\hline 46 & & & & & & & 0 & & & & -1 & & & & 1 \\
\hline 35 & & & & & & & 0 & 1 & & & & -1 & & & \\
\hline
\end{tabular}

Table 4: The collapsed incidence matrix of the regular three-fold cover of $W_{2}$. 
The distance-three graph of the Foster graph is a three-fold regular cover of the distancethree graph of $L\left(W_{2}\right)$. In turn, the distance-three graph of $L\left(W_{2}\right)$ is equal to the incidence graph of the complement of $W_{2}$. So the distance-three graph of the Foster graph can be represented in a similar manner as weighted incidence matrix of the complement $\overline{W_{2}}$ of $W_{2}$. This matrix is given in Table 5 . Combining Tables 4 and 5 , we obtain Table 6. It

\begin{tabular}{|c||c|c|c|c|c|c|c|c|c|c|c|c|c|c|c|}
\hline & 12 & 14 & 13 & 16 & 15 & 12 & 12 & 14 & 14 & 16 & 15 & 16 & 15 & 13 & 13 \\
& 34 & 23 & 24 & 25 & 26 & 36 & 35 & 26 & 25 & 23 & 23 & 24 & 24 & 26 & 25 \\
& 56 & 56 & 56 & 34 & 34 & 45 & 46 & 35 & 36 & 45 & 46 & 35 & 36 & 45 & 46 \\
\hline \hline 56 & & & & 0 & 0 & 0 & 0 & 0 & 0 & 0 & 0 & 0 & 0 & 0 & 0 \\
\hline 34 & & 0 & 0 & & & 0 & 0 & -1 & 1 & -1 & 1 & 1 & -1 & 1 & -1 \\
\hline 12 & & 0 & 0 & 0 & 0 & & & 1 & -1 & 1 & -1 & -1 & 1 & -1 & 1 \\
\hline 14 & 0 & & 0 & -1 & 1 & 1 & -1 & & & 0 & 0 & 1 & -1 & -1 & 1 \\
\hline 23 & 0 & & 0 & 1 & -1 & -1 & 1 & 0 & 0 & & & -1 & 1 & 1 & -1 \\
\hline 24 & 0 & 0 & & -1 & 1 & -1 & 1 & -1 & 1 & 1 & -1 & & & 0 & 0 \\
\hline 13 & 0 & 0 & & 1 & -1 & 1 & -1 & 1 & -1 & -1 & 1 & 0 & 0 & & \\
\hline 25 & 0 & 1 & -1 & & 0 & -1 & 1 & 1 & & -1 & 0 & 1 & 0 & -1 & \\
\hline 16 & 0 & -1 & 1 & & 0 & 1 & -1 & 0 & 1 & & -1 & & 1 & 0 & -1 \\
\hline 15 & 0 & 1 & -1 & 0 & & 1 & -1 & -1 & 0 & 1 & & -1 & & 1 & 0 \\
\hline 26 & 0 & -1 & 1 & 0 & & -1 & 1 & & -1 & 0 & 1 & 0 & -1 & & 1 \\
\hline 36 & 0 & -1 & 1 & 1 & -1 & & 0 & -1 & & 1 & 0 & 1 & & -1 & 0 \\
\hline 45 & 0 & 1 & -1 & -1 & 1 & & 0 & 0 & -1 & & 1 & 0 & 1 & & -1 \\
\hline 46 & 0 & -1 & 1 & -1 & 1 & 0 & & 1 & 0 & -1 & & -1 & 0 & 1 & \\
\hline 35 & 0 & 1 & -1 & 1 & -1 & 0 & & & 1 & 0 & -1 & & -1 & 0 & 1 \\
\hline
\end{tabular}

Table 5: The collapsed incidence matrix of the regular three-fold cover of $\overline{W_{2}}$.

remains to observe that this table effectively defines a $\mathcal{P} \times \mathcal{L}$-matrix $W=\left(w_{P, l}\right)$ over $\mathbb{Z}_{3}$ where $\mathcal{P}$ and $\mathcal{L}$ are the point- and the line-set of $W_{2}$. Let $\mathcal{F}$ denote the set of flags of $W_{2}$. Using $W$, let us define an $\mathcal{F} \times \mathcal{F}$-matrix $A=\left(a_{\left(P_{1}, l_{1}\right),\left(P_{2}, l_{2}\right)}\right)$ over $\mathbb{Z}_{3}$ by setting

$$
a_{\left(P_{1}, l_{1}\right),\left(P_{2}, l_{2}\right)}:=w_{\left(P_{2}, l_{1}\right)} \cdot w_{\left(P_{1}, l_{2}\right)}^{-1} .
$$

By reindexing the rows and columns we obtain

Theorem 7.1. The matrix A constructed above is a Godsil-Hensel matrix $\operatorname{GHM}\left(\mathbb{Z}_{3}, 45\right.$, $3,12)$. That is, it gives rise to a regular $(45,3,12)$-cover of $K_{45}$ with voltage group $\mathbb{Z}_{3}$.

Proof. This was checked using a computer.

Remark 7.2. i) The regular $(45,3,12)$-cover $\Gamma^{A}$ has 135 vertices. These vertices may be identified with the edges of the Foster graph, or, equivalently, with the flags of $\widetilde{W}_{2}$.

ii) Its automorphism group coincides with the automorphism group of the Foster graph, which is again isomorphic to the automorphism group of the regular $(36,3,12)$-cover constructed in Section 6.1. 


\begin{tabular}{|c||c|c|c|c|c|c|c|c|c|c|c|c|c|c|c|}
\hline & 12 & 14 & 13 & 16 & 15 & 12 & 12 & 14 & 14 & 16 & 15 & 16 & 15 & 13 & 13 \\
& 34 & 23 & 24 & 25 & 26 & 36 & 35 & 26 & 25 & 23 & 23 & 24 & 24 & 26 & 25 \\
& 56 & 56 & 56 & 34 & 34 & 45 & 46 & 35 & 36 & 45 & 46 & 35 & 36 & 45 & 46 \\
\hline \hline 56 & $\mathbf{0}$ & $\mathbf{0}$ & $\mathbf{0}$ & 0 & 0 & 0 & 0 & 0 & 0 & 0 & 0 & 0 & 0 & 0 & 0 \\
\hline 34 & $\mathbf{0}$ & 0 & 0 & $\mathbf{0}$ & $\mathbf{0}$ & 0 & 0 & -1 & 1 & -1 & 1 & 1 & -1 & 1 & -1 \\
\hline 12 & $\mathbf{0}$ & 0 & 0 & 0 & 0 & $\mathbf{0}$ & $\mathbf{0}$ & 1 & -1 & 1 & -1 & -1 & 1 & -1 & 1 \\
\hline 14 & 0 & $\mathbf{0}$ & 0 & -1 & 1 & 1 & -1 & $\mathbf{0}$ & $\mathbf{0}$ & 0 & 0 & 1 & -1 & -1 & 1 \\
\hline 23 & 0 & $\mathbf{0}$ & 0 & 1 & -1 & -1 & 1 & 0 & 0 & $\mathbf{0}$ & $\mathbf{0}$ & -1 & 1 & 1 & -1 \\
\hline 24 & 0 & 0 & $\mathbf{0}$ & -1 & 1 & -1 & 1 & -1 & 1 & 1 & -1 & $\mathbf{0}$ & $\mathbf{0}$ & 0 & 0 \\
\hline 13 & 0 & 0 & $\mathbf{0}$ & 1 & -1 & 1 & -1 & 1 & -1 & -1 & 1 & 0 & 0 & $\mathbf{0}$ & $\mathbf{0}$ \\
\hline 25 & 0 & 1 & -1 & $\mathbf{0}$ & 0 & -1 & 1 & 1 & $\mathbf{1}$ & -1 & 0 & 1 & 0 & -1 & $-\mathbf{1}$ \\
\hline 16 & 0 & -1 & 1 & $\mathbf{0}$ & 0 & 1 & -1 & 0 & 1 & $-\mathbf{1}$ & -1 & $\mathbf{1}$ & 1 & 0 & -1 \\
\hline 15 & 0 & 1 & -1 & 0 & $\mathbf{0}$ & 1 & -1 & -1 & 0 & 1 & $\mathbf{1}$ & -1 & $-\mathbf{1}$ & 1 & 0 \\
\hline 26 & 0 & -1 & 1 & 0 & $\mathbf{0}$ & -1 & 1 & $-\mathbf{1}$ & -1 & 0 & 1 & 0 & -1 & $\mathbf{1}$ & 1 \\
\hline 36 & 0 & -1 & 1 & 1 & -1 & $\mathbf{0}$ & 0 & -1 & $-\mathbf{1}$ & 1 & 0 & 1 & $\mathbf{1}$ & -1 & 0 \\
\hline 45 & 0 & 1 & -1 & -1 & 1 & $\mathbf{0}$ & 0 & 0 & -1 & $\mathbf{1}$ & 1 & 0 & 1 & $-\mathbf{1}$ & -1 \\
\hline 46 & 0 & -1 & 1 & -1 & 1 & 0 & $\mathbf{0}$ & 1 & 0 & -1 & $-\mathbf{1}$ & -1 & 0 & 1 & $\mathbf{1}$ \\
\hline 35 & 0 & 1 & -1 & 1 & -1 & 0 & $\mathbf{0}$ & $\mathbf{1}$ & 1 & 0 & -1 & $\mathbf{- 1}$ & -1 & 0 & 1 \\
\hline
\end{tabular}

Table 6: The combined collapsed incidence matrices.

iii) The cover is not distance transitive. Its automorphism group acts transitively of rank 10 on the vertices.

iv) Though, the construction seems to be rather generic, this is the only graph that we know, that can be obtained in this way. At the moment it seems that the existence of further examples of similar antipodal covers of $K_{n}$ depends on the knowledge of collinearity graphs of generalized quadrangles that possess regular distance regular covers.

v) Note the close relation between the construction of regular covers described in the beginning of this paper and the construction related to the Foster graph. In the former we construct regular covers from regular distance regular covers of $K_{n, n}$. Note that $K_{n, n}$ is the collinearity graph of a generalized di-gon. This is the degenerate case of a generalized $n$-gon. On the other hand, the latter construction starts out from a regular distance regular cover of the collinearity graph of a generalized quadrangle, and produces a regular cover of a complete graph on the flags.

\section{Spinoff observations}

\subsection{Regular Covers of class II}

Suppose that $H$ is a $\operatorname{GHM}\left(T, n, r, c_{2}\right)$ for $n-r c_{2}=2$ (i.e. $\delta=0$ ). Then, by Theorem 4.2, $H$ fulfills

$$
H^{2}=H H^{*}=(n-1) I+c_{2} \underline{T}(J-I) .
$$




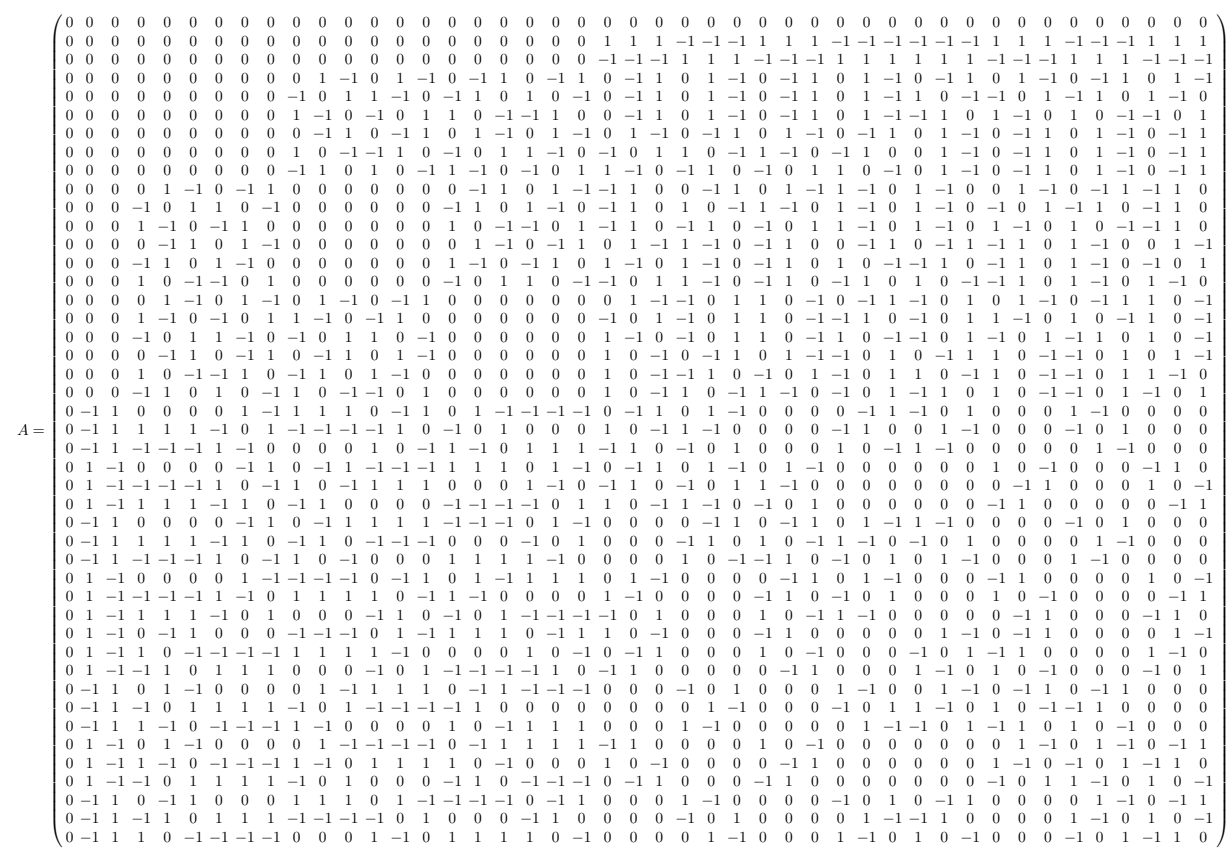

Table 7: $\mathrm{A} \operatorname{GHM}\left(\mathbb{Z}_{3}, 45,3,12\right)$.

Thus, the class of such Godsil-Hensel matrices $\operatorname{GHM}\left(T, n, r, c_{2}\right)$ coincides with the class of (non-degenerate) self-adjoint generalized conference matrices $\mathrm{GC}\left(T, c_{2}\right)$ (cf. [18, V.6.2] for details about generalized conference matrices).

Note that conference matrices are exactly the generalized conference matrices over the cyclic group of order 2 . Thus a conference matrix will be self-adjoint if and only if it is symmetric. For example, Taylor graphs that are locally a Paley graph, can be obtained from the Paley conference matrices.

\subsection{Regular Covers of class III}

Since we successfully could link regular covers of classes I and II to generalized Hadamard matrices and generalized conference matrices, respectively, it is natural to ask whether there are such connections also for regular covers of class III. According to Lemma 5.3, the Godsil-Hensel matrix $A$ of a cover with $\delta=2$ has to fulfill

$$
(A+I)^{2}=n I+4 A+c_{2} \underline{T}(J-I) .
$$

This is going to be the case if and only if

$$
(A-I)^{2}=n I+c_{2} \underline{T}(J-I) .
$$

If now $H$ is a symmetric Hadamard matrix of order $n>4$ with identity diagonal, then $-H+I$ is a $\operatorname{GHM}\left(\mathbb{Z}_{2}, n, 2,(n-4) / 2\right)$. Thus, every symmetric Hadamard matrix with 
constant diagonal gives rise to a distance regular double cover of a complete graph with $\delta=2$ (i.e., of class III). ${ }^{6}$

\section{Summary of new results}

In the previous sections relations of regular covers of complete graphs to generalized Hadamard matrices and generalized conference matrices were described. Using the known infinite families of such matrices, and the construction from Theorem 5.6, this implies the existence of several multiparameter families of regular covers of complete graphs. Next to the obvious benefit of obtaining a lot of interesting regular covers of complete graphs, this allows us to answer two open questions posed by Ch. Godsil in [44]:

i) In $[44, \S 9 . \mathrm{C}($ Question 4$)]$ it is asked whether there exist regular $\left(n, r, c_{2}\right)$ covers for which $n=r \cdot c_{2}$ (i.e. $\delta=-2$ ) and where $n$ is not a prime power. The regular $(36,3,12)$ cover that was described above gives a positive answer to this question. Since whenever $\delta=-2$ we have that $n$ is a square, this cover has $n$ as small as possible. Studying the literature about Hadamard matrices, we found that there exist many symmetric Hadamard matrices of order 36 with constant diagonal (cf. [102]). Without loss of generality we can assume that the diagonal is filled with the neutral element of $\mathbb{Z}_{2}$ (i.e. with 1 if we consider it in multiplicative notation as it is usual in the theory of Hadamard matrices, or with 0 if we write it in additive notation as it is usual in the theory of generalized Hadamard matrices). Switching to the language of generalized Hadamard matrices, we obtain skew generalized Hadamard matrices $\mathrm{gH}\left(\mathbb{Z}_{2}, 36\right)$. As was proved in Corollary 5.4, these matrices provide also Godsil-Hensel matrices $\operatorname{GHM}\left(\mathbb{Z}_{2}, 36,2,18\right)$ and thus regular $(36,2,18)$-covers. These also provide the smallest possible regular covers with $\delta=-2$ for which $n$ is not a prime power. However, none of them can be obtained using the construction given in Theorem 5.6, since there is no Hadamard matrix of order 6 . As an additional note, by [82], symmetric Bushtype Hadamard matrices of order $4 n^{4}$ exist for all odd $n$. Thus there exist regular $\left(4 n^{4}, 2,2 n^{4}\right)$ covers (with $\left.\delta=-2\right)$ for all odd $n$.

ii) In $\left[44, \S 9 . \mathrm{C}\left(\right.\right.$ Question 3)] it is asked whether there exist distance regular $\left(n, r, c_{2}\right)$ covers with $\delta=n-2-r c_{2}=2$ and such that $n$ is divisible by an odd prime. Using the obervations made in Section 8.2, the above mentioned symmetric Hadamard matrices of order 36 with constant diagonal that were constructed in [102] lead (by the observations made in Section 8.2) to 227 pairwise non-isomorphic regular $(36,2,16)$ covers. Moreover, the infinite series of symmetric Bush-type Hadamard matrices of order $4 n^{4}$ for odd $n$ that was constructed in [82], gives rise to an infinite series of $\operatorname{GHM}\left(\mathbb{Z}_{2}, 4 n^{4}, 2,2\left(n^{4}-1\right)\right)$ and thus to an infinite series of regular $\left(4 n^{4}, 2,2\left(n^{4}-\right.\right.$ 1))-covers (with $\delta=2$ ) for all odd $n$.

Let us now collect and discuss the data about the parameter families of regular covers that can be obtained by the constructions described in the previous sections. We may identify four potential sources of regular covers:

1. The regular $(45,3,12)$-cover on 135 vertices with $\delta=7$.

\footnotetext{
${ }^{6}$ Recall that $H-I$ is also a Godsil-Hensel matrix. However, in this case we have $\delta=-2$; i.e. we get a double cover of class II.
} 
2. Regular covers of type I (i.e. with $\delta=-2$ ) from skew generalized Hadamard matrices,

3. Regular covers of type II (i.e. with $\delta=0$ ) from self-adjoint generalized conference matrices,

4. Regular covers of type III (i.e. with $\delta=2$ ) from symmetric Hadamard matrices with identity diagonal,.

\subsection{About source 1}

The $(45,3,12)$-cover is certainly of a special interest. Even though the method by which it was constructed seems quite generic, all our efforts until now did not yield to a generalization into an infinite family. The involvement of the Foster graph at the core of the construction may indicate that this regular cover is sporadic. Certainly, it will deserve a special independent treatment at a forthcoming publication.

\subsection{About source 2}

This seems to be the most significant source of new examples in the current paper: A characterization by skew generalized Hadamard matrices plus a construction of skew generalized Hadamard matrices from arbitrary generalized Hadamard matrices. Thus, every known generalized Hadamard matrix gives rise to an infinite family of distance regular antipodal covers of complete graphs.

In the literature several infinite families of generalized Hadamard matrices can be found. Many of them are conveniently collected in [18, Sec. V.5], together with further references. In Table 8 we describe the parameter families of regular covers that arise from known parameter families of generalized Hadamard matrices over elementary abelian groups. The data about the generalized Hadamard matrices is taken from [18, Tab. V.5.10]. The above described parameter families of regular covers have some intersections with

\begin{tabular}{l|l|l}
\hline parameters of $H$ & $\left(n, r, c_{2}\right)$ & conditions \\
\hline $\mathrm{gH}\left(E_{p^{n}}, p^{m}\right)$ & $\left(p^{m 2^{t}}, p^{n}, p^{m 2^{t}-n}\right)$ & $m \geq n \geq 1, p$ prime, $t>0$ \\
\hline $\mathrm{gH}\left(E_{p^{n}}, 2 p^{m}\right)$ & $\left(2^{2^{t}} p^{m 2^{t}}, p^{n}, 2^{2^{t}} p^{m 2^{t}-n}\right)$ & $m \geq n \geq 1, p$ prime, $t>0$ \\
\hline $\mathrm{gH}\left(E_{p^{n}}, 4 p^{m}\right)$ & $\left(4^{2^{t}} p^{m 2^{t}}, p^{n}, 4^{2^{t}} p^{m 2^{t}-n}\right)$ & $m \geq n \geq 1, p$ prime, $t>0$ \\
\hline $\mathrm{gH}\left(E_{q}, 8 q\right)$ & $\left(8^{2^{t}} q^{2^{t}}, q, 8^{2^{t}} q^{2^{t}-1}\right)$ & $19<q<200, q$ prime power, \\
& & $t>0$ \\
\hline $\mathrm{gH}\left(E_{p}, 8 p\right)$ & $\left(8^{2^{t}} p^{2^{t}}, p, 8^{2^{t}} p^{2^{t}-1}\right)$ & $19<p, p$ prime, $t>0$ \\
\hline $\mathrm{gH}\left(E_{q}, k q\right)$ & $\left(k^{2^{t}} q^{2^{t}}, q, k^{2^{t}} q^{2^{t}-1}\right)$ & $q>\left((k-2) 2^{k-2}\right)^{2}$, \\
& & $\exists$ Hadamard matrix of order $k$ \\
& & $t>0$ \\
\hline
\end{tabular}

Table 8: Known families of gH-matrices.

known parameter families given in Table 1. Following we describe some such intersections: 
- If we put $t=1$ in the first row of Table 8 , then we obtain the parameters of the Godsil-Hensel construction in Table 1.

- If we put $m>n$, and $p=2$ in the second row of Table 8 , then we obtain parameters from Remark 3.2(iii).

- If we put $p=2$ in the third row of Table 8 , then we obtain parameters from Remark 3.2(iii).

Note that we did not fulfill an exhaustive search for intersections with known parameter families. In particular, the known parameter families of classical Hadamard matrices are not reflected in the table given above.

Let us come now to the "small examples" in Section 6. The restriction to generalized Hadamard matrices of order $\leq 15$ is motivated by the fact that all generalized Hadamard matrices of of these orders are known (cf. [40]). Of course, meanwhile also all generalized Hadamard matrices of order 16 were classified (cf. [41]). However, for this order too many matrices arise to give each of them a special attention. Moreover the range of orders $\leq 15$ already provides several interesting examples. To the best of our knowledge, the covers corresponding to rows \#6, \#13, \#15, \#16, and \#19 of Table 2, are new. The $(144,2,72)$ cover corresponding to row \#17 of Table 2 is a Taylor graph and is known. It seems that the non distance transitive covers with parameters $(81,9,9)$, and $(81,3,27)$ corresponding to rows \#11, and \#12 of Table 2, respectively, did not yet appear in the literature.

Following we show how the small examples fit into the parameter families given above in Table 8:

- \#6 is a $\mathrm{gH}\left(E_{3}, 6\right)$. It fits into the second family with $m=n=1, p=3$.

- \#11 is a $\mathrm{gH}\left(E_{9}, 9\right)$. It fits into the first family with $p=3, m=n=2$.

- \#12 is a $\mathrm{gH}\left(E_{3}, 9\right)$. It fits into the first family with $n=1, m=2, p=3$.

- \#13 is a $\mathrm{gH}\left(E_{5}, 10\right)$. It fits into the second family with $m=n=1, p=5$.

- \#15 is a $\operatorname{gH}\left(E_{3}, 12\right)$. These parameters fit into the third family with $n=m=1$, $p=3$.

- \#16 is a $\mathrm{gH}\left(E_{4}, 12\right)$. These parameters do not fit into any of the parameter families given above.

- \#17 is a $\mathrm{gH}\left(E_{2}, 12\right)$. This is a Paley Hadamard matrix.

- \#19 is a $\mathrm{gH}\left(E_{7}, 14\right)$. It fits into the second family with $m=n=1, p=7$.

\subsection{About source 3}

It seems that the connection between regular covers of type II and self-adjoint generalized conference matrices described in Section 8.1 did not yet appear in the literature. See Section 10.9 for extra discussion about the known sources of conference matrices.

\subsection{About source 4}

From every symmetric Hadamard matrix with constant diagonal one double cover of class II and one of class III may be obtained. These covers are closely related. The corresponding regular two-graphs are mutually complementary. We did not check whether the infinite series of symmetric Hadamard matrices given by Muzychuk and Xiang in [82] gives new examples of such graphs. 


\subsection{Taylor graphs}

Distance regular double covers of complete graphs are also known as Taylor graphs. They are very well studied not least because they are in a one to one correspondence with regular two-graphs. In this paper we get a number of families of regular double covers of complete graphs from Hadamard matrices and conference matrices. Every such regular cover is automatically a Taylor graph and thus locally a strongly regular graph with $k=2 \mu$. To our knowledge, the connection between symmetric Hadamard matrices and distance regular double covers of classes I and III was not yet observed in a general framework in literature. The same seems to hold also for the observation that there is a one to one correspondence between symmetric conference matrices and distance regular double covers of class II.

\section{Concluding discussion}

Starting from a single computer-aided observation about the existence of an antipodal cover of $K_{36}$ on 108 vertices, the current project (to the surprise of the authors) quickly developed into a much wider investigation on the intersection of diverse lines of algebraic graph theory, design theory, and finite geometries. The main body of this paper is naturally concerned with concrete results about antipodal graphs and new techniques for their construction. The extra dimension of the observed interdisciplinary links, are intentionally concentrated in the last section in form of remarks, bibliographical comments, historical reflections, hints, and even speculations.

\subsection{Antipodal covers}

Beginning from the seminal paper [99] and the background text [36], antipodal DRGs are playing a special role in the general theory. As we mentioned already earlier, together with [10], the thesis [66] remains the most significant comprehensive source of information about such graphs. Among the antipodal covers of complete graphs there are many interesting series and sporadic (known and putative) cases, which were not discussed in this paper, see e.g. [37], [42], [39], [43], [38]. Some of these graphs will be investigated more thoroughly in planned forthcoming publications.

\subsection{The matrix $\mathrm{gH}\left(\mathbb{Z}_{3}, 6\right)$}

In fact, the origin of the entire project goes back to the investigation of the primitive representation of the Hall-Janko group $J_{2}$ on 280 points. This action has rank 4 and subdegrees $1,36,108$, and 135. According to [61] (see also [30]) the 2-closure of this action coincides with $\operatorname{Aut}\left(J_{2}\right)$ and it has order $2^{8} \cdot 3^{3} \cdot 5^{2} \cdot 7$. Moreover, the graphs defined by the 2 -orbits of valency 36 and 135 both are strongly regular, non rank 3 graphs.

The orbital graph $\Gamma$ with parameters $(v, k, \lambda, \mu)=(280,36,8,4)$ is pseudogeometric with the parameters of the point graph of a generalized quadrangle $\operatorname{GQ}(9,3)$, though not geometric. It was noticed by Reichard in [86] that this graph is one of the few known exceptional strongly regular graphs, which satisfy the 4-vertex condition in the sense of [54]. Section 3.3 of [68] provides details about this graph ${ }^{7}$. The graph $\Gamma$ appears also in a number of other publications, e.g. [2], [63].

The stabilizer $H$ of a vertex in $\operatorname{Aut}\left(J_{2}\right)=\operatorname{Aut}(\Gamma)$ clearly has order $2^{5} \cdot 3^{3} \cdot 5$. Its abstract description is $\mathbb{Z}_{3} . S_{6} . S_{2}$, in other words $H$ is the extension of the exceptional triple

\footnotetext{
${ }^{7}$ We use this opportunity to correct a typo in the text there: the value of the parameter $\alpha$ should be 1 , not 0 .
} 
cover of $S_{6}$ (related to the famous Schur multiplier of the group $A_{6}$ ) with the exceptional outer automorphism of $S_{6}$, cf. [19]. The faithful action of $H$ on the suborbit of length 36 coincides with the full automorphism group of the well-known distance transitive graph with the intersection array $(6,5,4,1 ; 1,2,5,6)$, that is the antipodal cover of $K_{6,6}$, see [10]. The latter graph is also known as the incidence graph of the resolvable transversal design $\operatorname{RTD}_{2}(6,3)$ that appears from the famous hexacode, see e.g. [60, Sec. 1.9]. Finally, semibiplane (see Section 6.1) is one more name for this incidence structure.

The observation of all these properties of the distance transitive action of $H$ on 36 points created the initial motivation for the authors to investigate the two remaining faithful suborbital actions of $H$ of degree 108 and 135. As was already mentioned the joint use of COCO and GAP resulted in the discovery of two new antipodal distance regular covers of the complete graphs $K_{36}$, and $K_{45}$, respectively.

The generalized Hadamard matrix of order 6 played the role of a bridge from the single example on 108 points to an entirely new infinite class of similar objects. Paradoxically, it turned out to be very fortunate for the authors that this matrix was in a sense rediscovered by us in the course of finding a computer free description of the cover on 108 points.

In fact, the lucky role of the matrix $\mathrm{gH}\left(\mathbb{Z}_{3}, 6\right)$ as an "egg" for the antipodal cover on 36 points was observed in literature before, see e.g. [80].

Note also, that though the matrix $\mathrm{gH}\left(\mathbb{Z}_{3}, 6\right)$ may be regarded as the member of an infinite series of generalized Hadamard matrices, it has quite exceptional properties, provided one attempts to interpret it in suitable group-theoretical terms (see [34, Sec. 10]).

\subsection{The Foster graph and tilde geometries}

The Foster graph on 90 vertices, exploited in Section 7.3, derives its name from its discoverer R. M. Foster who started in the 1930s to collect small cubic symmetric graphs (cf. [32]). Apparently the example on 90 vertices appeared for the first time in 1966 in a manuscript by Foster. A census of his collection was published in [33]. In [99] the Foster graph was recognized as a 3-fold antipodal cover of the incidence graph of $W_{2}$. An explicit construction of the incidence structure $\widetilde{W}_{2}$, corresponding to the Foster graph, was given in [57]. A new interpretation of this structure in terms of diagram geometries was provided in [89]. An axiomatic description of a general class of such tilde geometries was developed in [59]. Many nice constructions of $\widetilde{W}_{2}$ may be found in [84].

\subsection{Another semibiplane}

The short note [62], written in a very concise style, still seems to be underrated in modern literature. Though the group-theoretical properties of the two semibiplanes presented there, remain hidden for the reader, we wish to believe that at least partial information was quite clear to the authors of [62]. We were very surprised to discover more and more new intriguing properties of these actions.

GAP identifies the automorphism group $F$ of the DRG, considered in Section 6.4 as $\left(\left(\left(\mathbb{Z}_{5} \times \mathbb{Z}_{5}\right): \mathbb{Z}_{5}\right): \mathbb{Z}_{8}\right): \mathbb{Z}_{2}$. It turns out that this group $F$ has up to equivalence a unique faithful transitive action on 50 points. Its rank is equal to 7 and it has subdegrees $1,2,2,5,10,10,20$, see [70, Sec. 11.4] for more details. It is 2-closed and coincides with the full automorphism group of the famous pentagon-pentagram decomposition of the Hoffman-Singleton graph, due to Robertson [88].

The mentioned action of $F$ on 50 points was identified in [51] in terms of the stabilizer 
of an edge in the flag graph of the unique projective plane of order 5 . In turn, this observation by P. Hafner was inspired by his careful analysis of the corresponding member of an infinite series of large graphs of diameter 2 discovered by McKay-Miller-Širáň [79], see also [50].

The group $F$ has a subgroup $K$ of index 2, which is nothing else but the automorphism group of the semibiplane $S=\operatorname{SBP}(50,10)$, considered in Section 6.4. The group $K$ acts transitively on the points and blocks of $S$. Both actions are similar (the action on the points is in fact 2-equivalent to the action of $F$ of degree 50). The 2-orbits of the intransitive action of $K$ on the points and blocks of $S$ form a Schurian coherent configuration $\mathfrak{M}$ with two fibres of size 50 and rank 26, with $\operatorname{Aut}(\mathfrak{M})=K$. COCO returns 79 merging association schemes of $\mathfrak{M}$, the rank varying from 3 to 13 . A few of these schemes are indeed remarkable. First, we get the transitive rank 13 action of the group $F$ on the vertices of the Levi graph $\Gamma$ of the $\operatorname{SBP}(50,10)$, with the subdegrees $1,2^{2}, 5^{3}, 10^{6}, 20$. The corresponding association scheme has in turn 50 merging association schemes (the biggest part of the above mentioned 79). There are two copies of a rank 5 metric non-Schurian scheme with the group $F$ as automorphism group, coming from the DRG $\Gamma$, two copies of a metric Schurian rank 5 scheme with valencies $1,7,15,35,42$ with the automorphism group isomorphic to Aut(HoSi), and a rank 3-scheme generated by the Higman-Sims graph (see e.g. [10]).

\subsection{From the Hadamard matrix of order 12 to 288 vertices}

The automorphism group $G$ of the antipodal cover \#17 in Table 2 has order 380160 . According to GAP this group is isomorphic to $\mathbb{Z}_{2} \times\left(M_{12}: \mathbb{Z}_{2}\right)$. Here the first $\mathbb{Z}_{2}$ corresponds to the transposition that acts semiregularly on the fibres (i.e., the voltage group), while the second $\mathbb{Z}_{2}$ corresponds to the outer automorphism of $M_{12}$. The group $G$ has rank 8 with subdegrees $1^{2}, 22^{2}, 55^{2}, 66^{2}$. The corresponding association scheme has two isomorphic metric mergings each of which provides the 2 -fold cover of $K_{144}$. The fact that the Mathieu group $M_{12}$ appears as a subgroup of $G$, can be explained in a slightly different context. Indeed, it was already known to M. Hall [52] that the automorphism group of the unique Hadamard matrix of order 12 is isomorphic to $\mathbb{Z}_{2} \cdot M_{12}$.

The group $M_{12}$ has two non-isomorphic transitive actions of degree 144 , both on the cosets of subgroups isomorphic to $\operatorname{PSL}(2,11)$. These actions were discussed in the literature, see e.g. [12]. The existence of two actions implies in a natural way the existence of a coset geometry with 144 points and 144 blocks, which turns out to be a semibiplane. The Levi graph $L$ of this semibiplane is a bipartite DRG with the parameters $(12,11,10,7 ; 1,2,5,12)$. The graph $L$ was constructed by Leonard in his Ph.D. thesis [73]. We have that $\operatorname{Aut}(L)=\operatorname{Aut}\left(M_{12}\right)=M_{12}: \mathbb{Z}_{2}$ has two orbits on the set of vertices. Brouwer proved that the Leonard graph $L$ is uniquely determined by its parameters. The initial proof appeared as a preprint in 1983, while a regular publication is [9].

I. V. Chuvaeva and A. A. Ivanov discovered the graph $L$ independently [15] (cf. [16]), becoming aware of the results of Leonard and Brouwer upon the completion of their project. In the approach developed in [15], they also constructed the point graph of the semibiplane which turns out to be a strongly regular graph $\Delta$ on 144 vertices, where the vertices may be identified with the cells of the Hadamard matrix of order 12. Moreover, they generalized the construction to arbitrary Hadamard matrices, proving that from every Hadamard matrix of order $4 m$ an SRG with parameters $\left(16 m^{2}, 8 m^{2}-2 m, 4 m^{2}, 4 m^{2}\right)$ may be constructed. The existence of this SRG, in turn, is equivalent to the existence of 
symmetric design. The latter design appears from a Hadamard matrix of order $16 \mathrm{~m}^{2}$ (cf. [47], [52]).

The SRG $\Delta$ has parameters $(v, k, \lambda, \mu)=(144,66,30,30)$, it was also constructed in [61], cf. [30], $\operatorname{Aut}(\Delta) \cong \operatorname{Aut}\left(M_{12}\right)$. It turns out that $\Delta$ belongs to a regular two-graph on 144 vertices (see also the general discussion in Section 10.8). Such a regular twograph naturally and canonically defines another SRG $\Sigma$ on 143 vertices with the parameters $(143,70,33,35)$, note that here $k=2 \mu$; while $\operatorname{Aut}(\Sigma)$ has order 1320 and is isomorphic to $\operatorname{PGL}(2,11)$. The standard double extension of $\Sigma$ (see again Section 10.8) coincides with the antipodal cover $\Gamma$. Though the existence of $\Gamma$ is the consequence of already known facts, it seems that such a parallel discussion of the two DRGs on 288 vertices related to $M_{12}$ did not appear in the literature before.

Last but not least, one more graph on 288 vertices, the Levi graph of $\operatorname{RTD}_{6}(12,2)$, generates a Schurian, symmetric imprimitive rank 7 association scheme with the valencies $1,1,22^{2}, 55^{2}, 132$. It deserves to be mentioned in the list of nice representations of decorations of $M_{12}$.

\subsection{More about the non-classical $\mathrm{gH}\left(\mathbb{Z}_{3}, 9\right)$}

Let us provide some more details regarding the second (non-classical) distance regular $(81,3,27)$-cover $\Gamma$ and the diverse structures and groups related to it. Recall once more that starting from a computer algebra experiment using COCO and GAP (resulting in the discovery of two hitherto unknown antipodal DRGs of order 108 and 135, respectively), a lot of further examples were discovered in a newly established standard way, using generalized Hadamard matrices. For many of the thus found concrete DRGs additional computer experiments may reveal new quite interesting features that in future may lead to further constructions of interesting combinatorial objects. We will use the graph $\Gamma$ of order 243 to show such possibilities and simultaneously to illustrate the ongoing difficulties involved in such activities.

First, let us consider the incidence structure $S=(P, B)=\operatorname{RTD}_{3}(9,3)$ with 27 points and 27 blocks that is defined by the non-classical $\operatorname{gH}\left(\mathbb{Z}_{3}, 9\right)$. Let $K=\operatorname{Aut}(S)$. As was already mentioned before, the order of $K$ is equal to 2916 (in fact, this order was already calculated in [77]). With the help of GAP a subgroup $H=\mathbb{Z}_{9} \times \mathbb{Z}_{3}$ of $K$ was found, that acts regularly on both sets $P$ and $B$. From this follows that in order to describe $S$, it is enough to provide just one block $b$, and to develop it using $H$. For example, we can take $b=\{(0,0),(1,0),(2,0),(3,0),(4,1),(5,2),(6,0),(7,2),(8,1)\}$. In fact, the set $b$ is a relative difference set over $H$ with respect to $\mathbb{Z}_{3}$ (see [18] for a definition). Having found $b$ with the aid of GAP, we later on were glad to observe $b$ in [74, Ex. on p. 518] as a particular case of a general construction of $\left(p^{2}, p, p^{2}, p\right)$-difference sets over $\mathbb{Z}_{p^{2}} \times \mathbb{Z}_{p}$, where $p$ is any odd prime power.

The group $K$ can be described in terms of a factorization $K=H \cdot K_{0}$ (into two subgroups with trivial intersection), where $K_{0}$ is the stabilizer of a point in $K$. Note that $K_{0}$ has order 108 . The group $K_{0}$ is well-known as the automorphism group of the Pappus configuration with 9 points and 9 lines (which is obtained from the affine plane of order 3 by removing one of the parallel classes of lines), see also [20].

On the next stage let us consider an auxiliary graph $\Sigma$, namely the Levi graph of the above defined incidence structure $S$, which has 54 vertices, and which is regular of valency 9 (therefore it has 243 edges, that is flags of $S$ ). Note that $\Sigma$ is a bipartite and antipodal DRG of diameter 4 with intersection array $(9,8,6,1 ; 1,3,8,9)$, cf. [10, p. 425]. Since $S$ is 
self-dual, we have that $|\operatorname{Aut}(\Sigma)|=2 \cdot|\operatorname{Aut}(S)|=5832$. The group $L=\operatorname{Aut}(\Sigma)$, too, may be represented by a factorization $\bar{H} \cdot L_{0}$, where $L_{0} \cong K_{0}$ again is the stabilizer of a point in $L$, while $\bar{H}$ is a natural subdirect product of the groups $D_{9}$ and $D_{3}$ of order 18 and 6 , respectively, that is obtained from the homomorphisms of both groups onto a group of order 2 .

Finally, it is possible to provide some information, strictly related to the graph $\Gamma$ and the group $M=\operatorname{Aut}(\Gamma)$. The group $K$ of order 2916 acts transitively on the set $F$ of flags of $S$ as a rank 39 permutation group, where also $K \unlhd M$. The quotient group $M / K$ appears as complement of $K$ in $M$, where $K / M \cong \mathbb{Z}_{3} \times S_{3}$. Thus $M=K:\left(\mathbb{Z}_{3} \times S_{3}\right)$ is a group of order 52488 , which has rank 12 and subdegrees $1,2,4^{2+1 \times 2}, 8,36^{2+1 \times 2}, 72$.

Note that $M$ can be obtained easily from the (faithful transitive) action of $L$ on the set $F$ of flags which has rank 22 :

- find the normalizer $N$ of $L$ in $\operatorname{Sym}(F)$ which has order $3 \cdot 5832$;

- get the 2-closure $N^{(2)}$, which coincides with $M$.

Note also that $M$ contains a unique normal subgroup isomorphic to $\mathbb{Z}_{3}$, which is inherited from the embedding $H=\mathbb{Z}_{9} \times \mathbb{Z}_{3}$ into $K$, and after that through $L$ into $M$. This subgroup Id $\times \mathbb{Z}_{3}$ of $H$ plays finally the role of the voltage group of the new antipodal graph $\Gamma$.

COCO returns 35 merging association schemes of the scheme of 2-orbits of the permutation group $(M, F)$, one of these schemes of rank 4 with valencies $1,2,80,160$ is the metric scheme $\mathfrak{M}$ generated by the graph $\Gamma$.

It turns out that there is an alternative, in a sense more attractive way to explain the origin of $\mathfrak{M}$. This way appears in the framework and spirit of concepts and techniques, introduced in [69], via the use of COCO-II. This time let us start from the Schurian association scheme $\mathfrak{N}_{1}$ consisting of the 2-orbits of the permutation group $(L, F)$ of rank 22.

- Compute $\operatorname{AAut}\left(\mathfrak{N}_{1}\right) \cong \mathbb{Z}_{3}$.

- Get the (unique) algebraic merging of $\mathfrak{N}_{1}$ with respect to $\mathbb{Z}_{3}$; the resulting scheme $\mathfrak{N}_{2}$ is again Schurian. It has rank 12 and (as it follows from the previous information) we have

$$
\mathfrak{N}_{2}=(2-\operatorname{orb}(M, F), F) \text {. }
$$

- Compute AAut $\left(\mathfrak{N}_{2}\right)=\mathbb{Z}_{2}$ (note that $\mathbb{Z}_{2}$ is generated by a proper algebraic automorphism of $\left.\mathfrak{N}_{2}\right)$.

- Get the (unique) algebraic merging of $\mathfrak{N}_{2}$ with respect to $\mathbb{Z}_{2}$; the resulting scheme $\mathfrak{N}_{3}$ this time is non-Schurian and has rank 10.

- Compute AAut $\left(\mathfrak{N}_{3}\right)=\mathbb{Z}_{2}$, note that again this group is generated by a proper algebraic automorphism.

- Once more get the (unique) algebraic merging of $\mathfrak{N}_{3}$ with respect to $\mathbb{Z}_{2}$. The resulting scheme $\mathfrak{N}_{4}$ is again non-Schurian and has rank 6 .

- Observe that $\mathfrak{N}_{4}$ has valencies $1,2,8,16,72,144$. Using the tensor of structure constants of $\mathfrak{N}_{4}$, it is easy to check that merging the classes of valency 8 and 72 and of valency 16 and 144, respectively produces one more non-Schurian association scheme $\mathfrak{N}_{5}=\mathfrak{M}$, which is our desired metric association scheme, generated by the graph $\Gamma$. 
Finally, we wish to mention (in view of the fact observed in [74]), that $S=\operatorname{RTD}_{3}(9,3)$ appears to be the first member of an infinite series of transversal designs, constructed with the aid of relative difference sets. Thus the current subsection inspires possible natural generalizations, which hopefully will be the subject of a further publication.

\subsection{The concept of a gH matrix}

It seems that Butson in [13] was the first to come up with an evident formulation of a definition for the concept of generalized Hadamard matrices. In his formulation the elements of a complex generalized Hadamard matrix $H$ are arbitrary complex roots of unity and, as in the classical definition, it is required that $H$ fulfills the identity

$$
H H^{*}=n I,
$$

where $n$ denotes the order of $H$.

Because $H$ has a finite order, Butson's definition is equivalent to the modern notion of a generalized Hadamard matrix over a suitable finite cyclic group.

In the definition of complex Hadamard matrices by Turyn (cf. [108]), the elements of $H$ are limited to the set $\{ \pm 1, \pm i\}$, while typically, in the literature the notion of complex Hadamard matrices coincides with the notion Butson-type generalized Hadamard matrices (cf. [80], [90]).

One more possibility to generalize the notion of Hadamard matrices is touched in [17]. Surprisingly, already Sylvester and Hadamard themselves considered generalizations of Hadamard matrices going beyond Butson's definition, see [22] for a detailed historical background.

As was already mentioned above, the requirement that $A^{*} A=A A^{*}$ in Definition 5.1, may be omitted. This possibility was discussed in the literature for a long time, $\mathrm{cf}$. the review MR557071(81i:05037) by D. Jungnickel in the Math. Reviews on the paper [103]. A short and elegant proof in [21] refers to the quotient ring of the group ring where the generalized Hadamard matrix lives, and otherwise imitates the classical proof for Hadamard matrices.

A lot of interesting links between generalized Hadamard matrices and diverse combinatorial structures were observed for many decades, [96], and [27] serve as nice starting sample patterns.

The definition of generalized Hadamard matrices allows further generalizations, among them the concept of a balanced generalized weighing matrix (briefly BGW) seems to be the most significant one. While for certain special cases BGW matrices are definitely linked to antipodal DRGs (see below), the general situation is less clear.

As a single striking example we wish to compare the $\operatorname{GHM}\left(\mathbb{Z}_{3}, 45,3,12\right)$ that was constructed in Section 7 (beware, this is not a generalized Hadamard matrix!) with the sporadic BGW $(45,12,3)$ announced by R. Mathon in 1987, see [65, p. 22] (note the coincidence of the parameters for both kinds of matrices).

Mathon's matrix defines a divisible semiplane with 135 points and 135 lines, as it is presented by Mathon in [18, p. 731]. We compared the Levi graph of Mathon's semiplane with the Levi graph of the design defined by the $\operatorname{GHM}\left(\mathbb{Z}_{3}, 45,3,12\right)$. The automorphism groups and the corresponding (non-Schurian) coherent configurations differ essentially.

A more successful attempt to reveal a possible common origin of these two sporadic matrices will be very welcome. 


\subsection{Distance regular double covers of $K_{n}$}

This class of DRGs is closely linked with the concept of a two-graph. Two-graphs, in turn, have three relatively independent origins:

- the investigation of certain classes of 2-transitive permutation groups by G. Higman and later on by D. Taylor [105];

- the graph extension theorem by E. Shult [97, 98];

- switching of graphs in the sense of J. J. Seidel.

A two-graph $(V, \Theta)$ consists of a set $V$ of vertices and a collection $\Theta$ of so-called odd 3subsets of vertices, such that each 4-subset of $V$ contains an even number of triples from $\Theta$. A two-graph is called a regular two-graph if it is simultaneously a balanced block design. For more details we refer to the collection [92], which contains a number of significant papers by Seidel et al related to diverse facets of two-graphs and their links with other combinatorial structures.

For any graph $\Gamma=(V, E)$ the collection of all those 3 -subsets of $V$, containing an odd number of edges from $E$ provides a two-graph. Assume now that $\Gamma$ is an SRG with $k=2 \mu$, and suppose that $\Gamma^{\prime}$ is the graph obtained from $\Gamma$ by adjoining an isolated additional vertex. Then the two-graph that corresponds to $\Gamma^{\prime}$ is a regular two-graph, and moreover, any regular two-graph may be constructed in this manner.

Assume that $\Gamma=(V, E)$ is an arbitrary graph. Let us construct a graph $\bar{\Gamma}$ (known as Shult's double extension) as follows: Take two copies $V^{\prime}, V^{\prime \prime}$ of the vertex set $V$ of $\Gamma$, and take two additional vertices $v^{\prime}, v^{\prime \prime}$, that together form the vertex set of $\bar{\Gamma}$. The edges of $\bar{\Gamma}$ are $\left\{v^{\prime}, x^{\prime \prime}\right\},\left\{v^{\prime \prime}, x^{\prime}\right\}$, for all $x \in V$; all edges of $\Gamma$ on both copies $V^{\prime}, V^{\prime \prime} ;\left\{x^{\prime}, y^{\prime \prime}\right\}$, whenever $\{x, y\}$ is not an edge of $\Gamma$.

It is easy to check that if $\Gamma$ is an SRG with parameters $(v, k, \lambda, \mu)$, such that $k=2 \mu$, then $\bar{\Gamma}$ is a distance regular double cover of the complete graph $K_{v+1}$, and vice versa, any antipodal double cover of a complete graph appears in this way. This result was attributed in [5] to M. J. Hoare with the credits for coming up with the idea of the construction of $\bar{\Gamma}$ to Shult and Taylor. Finally one gets a natural bijection between the antipodal covers, originating from an SRG $\Gamma$, and the regular two-graphs corresponding to $\Gamma^{\prime}$.

This is why the investigation of the antipodal distance regular double covers of complete graphs is usually reformulated in terms of regular two-graphs. A significant class of such objects is related to conference matrices, see below. The main interest here is related to a few sufficient conditions to construct a regular two-graph with the aid of the intermediate use of a symmetric Hadamard matrix of order $16 t^{2}$ with a constant diagonal, see [47], [92, pp. 168-170]. In particular, an arbitrary Hadamard matrix of order $4 t$ may be used as a starting point to get a desired matrix of order $16 t^{2}$. In our eyes, a careful comparison of these classical constructions by Seidel et al with the one presented in this paper deserves further attention.

An interesting question is also the comparison of the groups $\operatorname{Aut}(\bar{\Gamma})$ and $\operatorname{Aut}(\Gamma)$. Clearly, the order of the first group is at least twice as large as $|\operatorname{Aut}(\Gamma)|$. First attempts to discuss cases when Aut $(\bar{\Gamma})$ is "essentially" larger may be traced to [92, pp. 237-244]. For more advanced recent investigations we refer to [104]. 


\subsection{Conference matrices}

A conference matrix (briefly $\mathrm{C}$-matrix) of order $n$ is a square matrix $C$ with zero diagonal and otherwise with entries \pm 1 that satisfies

$$
C C^{\top}=(n-1) I
$$

The name was coined by V. Belevich in 1950 and is related to their applications in network conference telephony. The book [92] is again a good source of classical results related to C-matrices.

Every C-matrix can be normalized and thus is defined by its core $S$, which turns out to be a symmetric or antisymmetric matrix of order $n-1$, provided $n \equiv 2$ or $n \equiv 0(\bmod 4)$, respectively. A symmetric core defines the \pm 1 adjacency matrix (also called Seidel-matrix) of an SRG, which satisfies $k=2 \mu$. Therefore $\mathrm{C}$-matrices are equivalent to a special case of antipodal distance regular double covers of complete graphs.

Any $\mathrm{C}$-matrix may be regarded as a special case of a BGW matrix, thus providing extra evidence of the significance of balanced matrices in the current context. Another interesting class of combinatorial structures, that coexist with conference matrices is presented in [48].

A more general particular case of BGW matrices is provided by generalized conference matrices (see [18] for precise definitions and basic properties). In particular, generalized C-matrices have essential links with projective planes (cf. [56]). Possibilities for using such matrices also for the construction of antipodal covers of $K_{n}$ are of a definite interest.

\subsection{The Brouwer-Godsil-Wilbrink text}

The text [11] is the draft of a paper in progress, that was created almost 20 years ago. Its intention was to investigate isomorphisms and non-isomorphisms of various antipodal DRGs of diameter three, constructed as covers of polar spaces of rank 1.

The style of the presentation is concise, all references appear implicitly, and the amount of the covered material is very huge. This text already influenced a number of publications. In particular a part of the new results from [11] are discussed in [42]. The links of covers on $q^{3}$ vertices with extended generalized quadrangles (in the sense of [14]) are of a special interest.

It may be very helpful to undertake a new wave of efforts in order to transform the draft [11] into a regular publication, which will also take into account the ongoing progress in the area.

\subsection{Miscellanea}

Generalized Hadamard matrices and related structures constitute a rapidly growing research area.

Below we wish to attract the reader's attention just to a few particular links of development which seem in our eyes on the frontier of possible significant interactions with antipodal covers. Interesting exceptional features of $\mathrm{gH}\left(\mathbb{Z}_{3}, 6\right)$, and possibilities for generalizations are discussed in [25] in a developed group theoretical setting.

A promising class of circulant generalized Hadamard matrices is produced in [7]. Further steps in a more general approach are outlined in [74].

One more class of generalized Hadamard matrices of order $n=m^{2}$ is studied in [53], in relation with projective planes of order $n$. 
Kharaghani and Torabi in [67] exploited BGW matrices for the construction of SRGs with a spread, which are equivalent to certain antipodal covers via Brouwer's construction (cf. Table 1). These covers are discussed in [71].

We also wish to mention numerous investigations in the area of semibiplanes and more general structures, with [111] as a sample paper.

\subsection{Further perspectives}

The project of the classification of all distance transitive graphs seems to be close to completion, cf. [58], [109], [1]. In particular, all antipodal distance transitive covers of complete graphs are classified (cf. [46]).

In comparison, the problem of the classification of all DRGs (even of diameter at least 3 ) is hopeless. In this fashion, the classification of antipodal distance regular covers of complete graphs turns out to be an essentially difficult ingredient of the more general case.

Indeed, it follows from [31], that there are numerous antipodal covers that appear via the deletion of a spread from a pseudo-geometric GQ $(n-1, n+1)$-graph. The same paper provides also a prolific construction of pseudo-geometric $\mathrm{GQ}(n+1, n-1)$-graphs for which it is not clear whether they contain spreads leading to antipodal covers. Sven Reichard, in the course of the preparation of his thesis [87], arranged a computer-experiment. Applying Fon Der Flaass' [31, Construction 1] to the affine plane of order 4, he generated a random sample of 1000 pseudo-geometric GQ $(5,3)$-SRGs. It turned out that all of the generated graphs were mutually non-isomorphic. However, none of them had a spread leading to a $(16,6,2)$-cover. We are aware of just one graph that can arise by applying [31, Construction 1] to the affine plane of order 4 and that gives rise to an antipodal $(16,6,2)$ cover on 96 vertices. This example, due to Muzychuk, is described in the end of [81, Section 5].

Thus the following research leads remain actual in the course of the investigation of antipodal distance regular covers of complete graphs:

- which feasible sets of parameters are realizable;

- for which parameter sets there exist prolific constructions (in the sense of [31]);

- description of the automorphism groups of known classes of graphs;

- isomorphisms between graphs sharing the same parameters.

As a quite practical immediate task a complete constructive enumeration of graphs with a "small" number of vertices seems to be very reasonable, cf. Table 3.3 in [66]. Note that according to a recent result in [72], each distance transitive graph admits a non-trivial semiregular automorphism. From this point of view, regular antipodal covers provide a nice compromise between two extremes: distance transitive graphs and distance regular graphs with trivial automorphism group.

We hope that a systematic search for new classes of Godsil-Hensel matrices which originate from generalized Hadamard matrices and their diverse generalizations will be getting a new surge of interest.

\section{Acknowledgments}

We are much grateful to Andries Brouwer, Chris Godsil, and Edwin van Dam for helpful comments and information. We thank Doug Leonard for the suggested numerous significant linguistical corrections, and Misha Muzychuk for helpful remarks. Last but not least, 
the long-standing collaboration with Sven Reichard, Andy Woldar and Matan Ziv-Av was very productive for the purposes of the research presented in the current text.

\section{References}

[1] M. R. Alfuraidan and J. I. Hall, Imprimitive distance-transitive graphs with primitive core of diameter at least 3, Michigan Math. J. 58 (2009), 31-77.

[2] B. Bagchi, A regular two-graph admitting the Hall-Janko-Wales group, Sankhyā Ser. A 54 (1992), 35-45, Combinatorial mathematics and applications (Calcutta, 1988).

[3] T. D. Bending and D. Fon-Der-Flaass, Crooked functions, bent functions, and distance regular graphs, Electron. J. Combin. 5 (1998), R\#34.

[4] T. Beth, D. Jungnickel and H. Lenz, Design theory. Vol. I, volume 69 of Encyclopedia of Mathematics and its Applications, Cambridge University Press, Cambridge, second edition, 1999.

[5] N. L. Biggs, Distance-regular graphs with diameter three, In Algebraic and geometric combinatorics, volume 65 of North-Holland Math. Stud., pages 69-80. North-Holland, Amsterdam, 1982.

[6] B. W. Brock, Hermitian congruence and the existence and completion of generalized Hadamard matrices, J. Combin. Theory Ser. A 49 (1988), 233-261.

[7] B. W. Brock, A new construction of circulant $\mathrm{GH}\left(p^{2} ; Z_{p}\right)$, Discrete Math. 112 (1993), 249-252.

[8] A. E. Brouwer, Distance regular graphs of diameter 3 and strongly regular graphs, Discrete Math. 49 (1984), 101-103.

[9] A. E. Brouwer, On the uniqueness of a regular thin near octagon on 288 vertices (or the semibiplane belonging to the Mathieu group $M_{12}$ ), Discrete Math. 126 (1994), 13-27.

[10] A. E. Brouwer, A. M. Cohen and A. Neumaier, Distance-regular graphs, volume 18 of Ergebnisse der Mathematik und ihrer Grenzgebiete. 3. Folge, Springer-Verlag, Berlin etc., 1989.

[11] A. E. Brouwer, C. D. Godsil and H. A. Wilbrink, Isomorphisms between antipodal distanceregular graphs of diameter three, Unpublished manuscript.

[12] F. Buekenhout, Geometries for the Mathieu group $M_{12}$, In Combinatorial theory (Schloss Rauischholzhausen, 1982), volume 969 of Lecture Notes in Math., Springer, Berlin, 1982, pages 74-85.

[13] A. T. Butson, Generalized Hadamard matrices, Proc. Amer. Math. Soc. 13 (1962), 894-898.

[14] P. J. Cameron, Covers of graphs and EGQs, Discrete Math. 97 (1991), 83-92.

[15] I. V. Chuvaeva and A. A. Ivanov, Action of the group $M_{12}$ on Hadamard matrices, In Investigations in the algebraic theory of combinatorial objects (Russian), Vsesoyuz. NauchnoIssled. Inst. Sistem. Issled., Moscow, 1985, pages 159-169.

[16] I. V. Chuvaeva and A. A. Ivanov, Action of the group $M_{12}$ on Hadamard matrices, In I. A. Faradžev, A. A. Ivanov, M. H. Klin, and A. J. Woldar, editors, Investigations in algebraic theory of combinatorial objects, volume 84 of Mathematics and its Applications (Soviet Series), Kluwer Academic Publishers Group, Dordrecht, 1994, pages 395-408. (English translation of [15]).

[17] J. H. E. Cohn, Hadamard matrices and some generalisations, Amer. Math. Monthly 72 (1965), 515-518. 
[18] C. J. Colbourn and J. H. Dinitz (eds.), Handbook of combinatorial designs, Discrete Mathematics and its Applications (Boca Raton), Chapman \& Hall/CRC, Boca Raton, FL, second edition, 2007.

[19] J. H. Conway, R. T. Curtis, S. P. Norton, R. A. Parker and R. A. Wilson, Atlas of finite groups, Oxford University Press, Eynsham, 1985, Maximal subgroups and ordinary characters for simple groups, With computational assistance from J. G. Thackray.

[20] H. S. M. Coxeter, The Pappus configuration and the self-inscribed octagon, I, Nederl. Akad. Wetensch. Proc. Ser. A 80=Indag. Math., 39 (1977), 256-269.

[21] R. Craigen and W. de Launey, Generalized Hadamard matrices whose transposes are not generalized Hadamard matrices, J. of Combin. Designs 17 (2009), 456-458.

[22] R. Craigen and R. Woodford, Power Hadamard matrices, Discrete Math. 308 (2008), 28682884.

[23] D. de Caen and D. Fon-Der-Flaass, Distance regular covers of complete graphs from Latin squares, Des. Codes Cryptogr. 34 (2005), 149-153.

[24] D. de Caen, R. Mathon and G. E. Moorhouse, A family of antipodal distance-regular graphs related to the classical Preparata codes, J. Algebraic Combin. 4 (1995), 317-327.

[25] W. de Launey, Generalised Hadamard matrices which are developed modulo a group, Discrete Math. 104 (1992), 49-65.

[26] C. Delorme, Regularité métrique forte, Rapport de Recherche No. 156, Univ. Paris sud, Orsay, 1983.

[27] P. Delsarte and J.-M. Goethals, Tri-weight codes and generalized Hadamard matrices, Information and Control 15 (1969), 196-206.

[28] D. A. Drake, Partial $\lambda$-geometries and generalized Hadamard matrices over groups, Canad. J. Math. 31 (1979), 617-627.

[29] I. Faradžev and M. Klin, Computer package for computations with coherent configurations, In Proc. ISSAC-91, pages 219-223, Bonn, 1991, ACM Press.

[30] I. A. Faradžev, M. H. Klin and M. E. Muzichuk, Cellular rings and groups of automorphisms of graphs, In I. Faradžev, A. Ivanov, M. Klin, and A. Woldar (eds), Investigations in algebraic theory of combinatorial objects, volume 84 of Math. Appl. (Soviet Ser.), Kluwer Acad. Publ., Dordrecht, 1994, pages 1-152.

[31] D. G. Fon-Der-Flaass, New prolific constructions of strongly regular graphs, Adv. Geom. 2 (2002), 301-306.

[32] R. M. Foster, Geometrical circuits of electrical networks, Trans. Amer. Inst. Elec. Engin. 51 (1932), 309-317.

[33] R. M. Foster, The Foster census, Charles Babbage Research Centre, Winnipeg, MB, 1988, R. M. Foster's census of connected symmetric trivalent graphs, With a foreword by H. S. M. Coxeter, With a biographical preface by Seymour Schuster, With an introduction by I. Z. Bouwer, W. W. Chernoff, B. Monson and Z. Star, Edited and with a note by Bouwer.

[34] J. C. Galati, A group extensions approach to relative difference sets, J. Combin. Des. 12 (2004), 279-298.

[35] The GAP Group, GAP - Groups, Algorithms, and Programming, Version 4.4.10, 2007.

[36] A. Gardiner, Antipodal covering graphs, J. Combinatorial Theory Ser. B 16 (1974), 255-273.

[37] A. Gardiner, Antipodal graphs of diameter three, Linear Algebra Appl. 46 (1982), 215-219.

[38] A. Gardiner, Distance-transitive antipodal covers: the extremal case, Discrete Math. 134 (1994), 63-64, Algebraic and topological methods in graph theory (Lake Bled, 1991). 
[39] A. D. Gardiner, C. D. Godsil, A. D. Hensel and G. F. Royle, Second neighbourhoods of strongly regular graphs, Discrete Math. 103 (1992), 161-170.

[40] P. B. Gibbons and R. Mathon, Signings of group divisible designs and projective planes, Australas. J. Combin. 11 (1995), 79-104.

[41] P. B. Gibbons and R. Mathon, Enumeration of generalized Hadamard matrices of order 16 and related designs, J. Combin. Des. 17 (2009), 119-135.

[42] C. D. Godsil, Krein covers of complete graphs, Australas. J. Combin. 6 (1992), 245-255.

[43] C. D. Godsil, Geometric distance-regular covers, New Zealand J. Math. 22 (1993), 31-38.

[44] C. D. Godsil, Covers of complete graphs, In Progress in algebraic combinatorics (Fukuoka, 1993), volume 24 of Adv. Stud. Pure Math., Math. Soc. Japan, Tokyo, 1996, pages 137-163.

[45] C. D. Godsil and A. D. Hensel, Distance regular covers of the complete graph, J. Combin. Theory Ser. B 56 (1992), 205-238.

[46] C. D. Godsil, R. A. Liebler and C. E. Praeger, Antipodal distance transitive covers of complete graphs, European J. Combin. 19 (1998), 455-478.

[47] J.-M. Goethals and J. J. Seidel, Strongly regular graphs derived from combinatorial designs, Canad. J. Math. 22 (1970), 597-614.

[48] M. Greig, H. Haanpää and P. Kaski, On the coexistence of conference matrices and near resolvable 2-(2k+1,k,k-1) designs, J. Combin. Theory Ser. A 113 (2006), 703-711.

[49] J. L. Gross and T. W. Tucker, Topological graph theory, Dover Publications Inc., Mineola, NY, 2001.

[50] P. R. Hafner, The Hoffman-Singleton graph and its automorphisms, J. Algebraic Combin. 18 (2003), 7-12.

[51] P. R. Hafner, On the graphs of Hoffman-Singleton and Higman-Sims, Electron. J. Combin. 11 (2004), R\#77.

[52] M. Hall, Jr, Group properties of Hadamard matrices, J. Austral. Math. Soc. Ser. A 21 (1976), 247-256.

[53] J. L. Hayden, Generalized Hadamard matrices, Des. Codes Cryptogr. 12(1):69-73, 1997.

[54] M. D. Hestenes and D. G. Higman. Rank 3 groups and strongly regular graphs. In Computers in algebra and number theory (Proc. SIAM-AMS Sympos. Appl. Math., New York, 1970), pages 141-159. SIAM-AMS Proc., Vol. IV. Amer. Math. Soc., Providence, R.I., 1971.

[55] K. J. Horadam, Hadamard matrices and their applications, Princeton University Press, Princeton, NJ, 2007.

[56] Y. J. Ionin and M. S. Shrikhande, Combinatorics of symmetric designs, volume 5 of New Mathematical Monographs, Cambridge University Press, Cambridge, 2006.

[57] T. Ito, Bipartite distance-regular graphs of valency three, Linear Algebra Appl. 46 (1982), 195-213.

[58] A. A. Ivanov, Distance-transitive graphs and their classification, In Investigations in algebraic theory of combinatorial objects, volume 84 of Math. Appl. (Soviet Ser.), Kluwer Acad. Publ., Dordrecht, 1994, pages 283-378.

[59] A. A. Ivanov, Geometry of sporadic groups I, Petersen and tilde geometries, volume 76 of Encyclopedia of Mathematics and its Applications, Cambridge University Press, Cambridge, 1999.

[60] A. A. Ivanov, The Monster group and Majorana involutions, volume 176 of Cambridge Tracts in Mathematics, Cambridge University Press, Cambridge, 2009. 
[61] A. A. Ivanov, I. A. Faradžev and M. H. Klin, The primitive representations of the non-abelian simple groups of order less than $10^{6}$, Part 2, Preprint, Institute for System Studies, Moscow, 1984, in Russian.

[62] Z. Janko and T. Van Trung, Two new semibiplanes, J. Combin. Theory Ser. A 33 (1982), $102-105$.

[63] L. K. Jørgensen and M. Klin, Switching of edges in strongly regular graphs, I, A family of partial difference sets on 100 vertices, Electron. J. Combin. 10 (2003), R\#17.

[64] D. Jungnickel, On difference matrices, resolvable transversal designs and generalized Hadamard matrices, Math. Z. 167 (1979), 49-60.

[65] D. Jungnickel, Balanced generalized weighing matrices and related structures, Quaderni Elettronici del Seminario di Geometria Combinatoria, 16E (Feb. 2005), 1-39, Università degli Studi di Roma "La Sapienza" — Dipartimento di Matematica.

[66] A. Jurišić, Antipodal covers, PhD thesis, University of Waterloo, Ontario, Canada, 1995.

[67] H. Kharaghani and R. Torabi, On a decomposition of complete graphs, Graphs Combin. 19 (2003), 519-526.

[68] M. Klin, M. Meszka, S. Reichard and A. Rosa, The smallest non-rank 3 strongly regular graphs which satisfy the 4-vertex condition, Bayreuth. Math. Schr. 74 (2005), 145-205.

[69] M. Klin, M. Muzychuk, C. Pech, A. Woldar and P.-H. Zieschang, Association schemes on 28 points as mergings of a half-homogeneous coherent configuration, European J. Combin. 28 (2007), 1994-2025.

[70] M. Klin, M. Muzychuk and M. Ziv-Av, Computer algebra experimentation with Higmanian rank 5 association schemes on 40 vertices and related combinatorial objects, Technical report, Ben Gurion University of the Negev, Beer Sheva, 2007, 91pp.

[71] M. Klin, S. Reichard and A. Woldar, Siamese combinatorial objects via computer algebra experimentation, In M. Klin, G. A. Jones, A. Jurišić, M. Muzychuk and I. Ponomarenko (eds.), Algorithmic Algebraic Combinatorics and Gröbner Bases, Springer, Berlin Heidelberg, 2009, pages 67-112.

[72] K. Kutnar and P. Šparl, Distance-transitive graphs admit semiregular automorphisms, Eur. J. Combin. 31 (2010), 25-28.

[73] D. A. Leonard, Semi-biplanes and semi-symmetric designs, PhD thesis, Ohio Staate University, 1980.

[74] S. L. Ma and A. Pott, Relative difference sets, planar functions, and generalized Hadamard matrices, J. Algebra 175 (1995), 505-525.

[75] M. Masuyama, On difference sets for constructing orthogonal arrays of index two and of strength two, Rep. Stat. Appl. Res. JUSE 5 (1957), 27-34.

[76] R. Mathon, 3-class association schemes, In Proceedings of the Conference on Algebraic Aspects of Combinatorics (Univ. Toronto, Toronto, Ont., 1975), Congressus Numerantium, No. XIII, Winnipeg, Man., 1975, Utilitas Math, pages 123-155.

[77] V. C. Mavron and V. D. Tonchev, On symmetric nets and generalized Hadamard matrices from affine designs, J. Geom. 67 (2000), 180-187, Second Pythagorean Conference (Pythagoreion, 1999).

[78] B. D. McKay, nauty User's Guide (Version 2.4), Department of Computer Science, Australian National University, Canberra, 2007.

[79] B. D. McKay, M. Miller and J. Širáň, A note on large graphs of diameter two and given maximum degree, J. Combin. Theory Ser. B 74 (1998), 110-118. 
[80] G. E. Moorhouse, The 2-transitive complex Hadamard matrices, Preprint, University of Wyoming, 2001, 32pp.

[81] M. Muzychuk, A generalization of Wallis-Fon-Der-Flaass construction of strongly regular graphs, J. Algebr. Comb. 25 (2007), 169-187.

[82] M. Muzychuk and Q. Xiang, Symmetric Bush-type Hadamard matrices of order $4 m^{4}$ exist for all odd m, Proc. Amer. Math. Soc. 134 (2006), 2197-2204.

[83] R. E. A. C. Paley, On orthogonal matrices, J. Math. Phys. 12 (1933), 311-320.

[84] A. Pasini and H. Van Maldeghem, Some constructions and embeddings of the tilde geometry, Note Mat. 21 (2002/03), 1-33.

[85] G. Pilz, Near-rings, The theory and its applications, volume 23 of North-Holland Mathematics Studies, North-Holland Publishing Co., Amsterdam, second edition, 1983.

[86] S. Reichard, A criterion for the $t$-vertex condition of graphs, J. Combin. Theory Ser. A 90 (2000), 304-314.

[87] S. Reichard, Computational and Theoretical Analysis of Coherent Configurations and Related Incidence Structures, PhD thesis, University of Delaware, 2003.

[88] N. Robertson, Graphs Minimal Under Girth, Valency, and Connectivity Constraints, $\mathrm{PhD}$ thesis, University of Waterloo, Waterloo, Ontario, 1969.

[89] M. A. Ronan and G. Stroth, Minimal parabolic geometries for the sporadic groups, European J. Combin. 5 (1984), 59-91.

[90] J. Seberry, Complex Hadamard matrices, Linear and Multilinear Algebra 1 (1973), 257-272.

[91] J. Seberry, A construction for generalized Hadamard matrices, J. Statist. Plann. Inference, 4 (1980), 365-368.

[92] J. J. Seidel, Geometry and combinatorics, Academic Press Inc., Boston, MA, 1991, Selected works of J. J. Seidel, Edited and with a preface by D. G. Corneil and R. Mathon.

[93] E. Seiden, On the problem of construction of orthogonal arrays, Ann. Math. Statistics 25 (1954), 151-156.

[94] S. A. Shad, Regular thin near $N$-gons and balanced incomplete block designs, Arabian J. Sci. Engrg. 9 (1984), 251-260.

[95] J. Shawe-Taylor, Coverings of complete bipartite graphs and associated structures, Discrete Math. 134 (1994), 151-160, Algebraic and topological methods in graph theory (Lake Bled, 1991).

[96] S. S. Shrikhande, Generalized Hadamard matrices and orthogonal arrays of strength two, Canad. J. Math. 16 (1964), 736-740.

[97] E. Shult, The graph extension theorem, Proc. Amer. Math. Soc. 33 (1972), 278-284.

[98] E. Shult, Supplement to "the graph extension theorem", Technical report, University of Florida, Gainesville, FL, 1972, 57pp.

[99] D. H. Smith, Primitive and imprimitive graphs, Quart. J. Math. Oxford Ser. (2) 22 (1971), 551-557.

[100] L. H. Soicher, The GRAPE package for GAP, Version 4.3, 2006.

[101] C. Somma, An infinite family of perfect codes in antipodal graphs, Rend. Mat. (7) 3 (1983), 465-474.

[102] E. Spence, Regular two-graphs on 36 vertices, Linear Algebra Appl. 226/228 (1995), 459497. 
[103] D. J. Street, Generalized Hadamard matrices, orthogonal arrays and $F$-squares, Ars Combin. 8 (1979), 131-141.

[104] D. B. Surowski, Automorphism groups of certain unstable graphs, Math. Slovaca 53 (2003), 215-232.

[105] D. E. Taylor, Regular 2-graphs, Proc. London Math. Soc. (3) 35 (1977), 257-274.

[106] D. E. Taylor and R. Levingston, Distance-regular graphs, In Combinatorial mathematics (Proc. Internat. Conf. Combinatorial Theory, Australian Nat. Univ., Canberra, 1977), volume 686 of Lecture Notes in Math., Springer, Berlin, 1978, pages 313-323.

[107] J. A. Thas, Two infinite classes of perfect codes in metrically regular graphs, J. Combinatorial Theory Ser. B 23 (1977), 236-238.

[108] R. J. Turyn, Complex Hadamard matrices, In Combinatorial Structures and their Applications (Proc. Calgary Internat. Conf., Calgary, Alta., 1969), Gordon and Breach, New York, 1970, pages 435-437.

[109] J. van Bon, Finite primitive distance-transitive graphs, European J. Combin. 28 (2007), $517-532$.

[110] H. Wielandt, Finite permutation groups, Translated from the German by R. Bercov. Academic Press, New York, 1964.

[111] P. Wild, Biaffine planes and divisible semiplanes, J. Geom. 25 (1985), 121-130. 